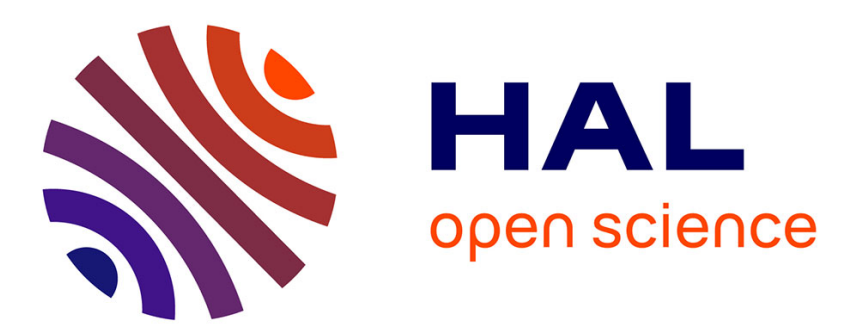

\title{
Implementation of multi-component Zhdanov closure in SOLEDGE3X
}

\author{
Hugo Bufferand, J Balbin, S Baschetti, J Bucalossi, G Ciraolo, A Gallo, R \\ Ghendrih, N Mao, P Rivals, H Tamain, et al.
}

\section{- To cite this version:}

Hugo Bufferand, J Balbin, S Baschetti, J Bucalossi, G Ciraolo, et al.. Implementation of multicomponent Zhdanov closure in SOLEDGE3X. 2021. hal-03243371v2

\section{HAL Id: hal-03243371 \\ https://hal.science/hal-03243371v2}

Preprint submitted on 28 Jul 2021

HAL is a multi-disciplinary open access archive for the deposit and dissemination of scientific research documents, whether they are published or not. The documents may come from teaching and research institutions in France or abroad, or from public or private research centers.
L'archive ouverte pluridisciplinaire HAL, est destinée au dépôt et à la diffusion de documents scientifiques de niveau recherche, publiés ou non, émanant des établissements d'enseignement et de recherche français ou étrangers, des laboratoires publics ou privés. 


\title{
Implementation of multi-component Zhdanov closure in SOLEDGE3X
}

\author{
H. Bufferand, J. Balbin, S. Baschetti, J. Bucalossi, G. Ciraolo, \\ Ph. Ghendrih, R. Mao, N. Rivals, P. Tamain, H. Yang \\ IRFM-CEA, F-13108 Saint-Paul-Lez-Durance, France \\ E-mail: hugo.bufferand@cea.fr
}

G. Giorgiani, F. Schwander, M. Scotto d'Abusco, E. Serre

Aix-Marseille Univ., CNRS, Centrale Marseille, M2P2, Marseille, France

J. Denis, Y. Marandet, M. Raghunathan

Aix-Marseille Univ., CNRS, PIIM, Marseille, France

P. Innocente

RFX, Padova, Italy

D. Galassi

Swiss Plasma Center, Lausanne, Switzerland

\begin{abstract}
.
The multi-component fluid closure derived by Zhdanov [1] is implemented in the fluid code SOLEDGE3X-EIRENE to deal with arbitrary edge plasma composition. The closure assumes no distinction in between species such as light vs heavy species separation. The work of Zhdanov is rewritten in a matricial form in order to clearly link friction forces and heat fluxes to the different species velocities and temperature gradients.
\end{abstract}

Submitted to: Plasma Phys. Control. Fusion

\section{Introduction}

In order to interpret experiments and in the perspective of predicting edge plasma properties for future nuclear fusion devices, a dedicated effort is made to simulate plasma-wall interaction. Edge plasma collisionality being moderate to high, a fluid description of the plasma can be considered as a good approximation, at least to estimate engineering quantities of interest such as heat load on the plasma facing components. 
More precisely, two-dimensional transport codes $[2,3,4]$ are used to provide in a few hours of computation an overall description of the edge plasma, the price of reduced computation time being a raw ad-hoc description of turbulent cross-field transport. Conversely, three-dimensional fluid codes $[5,6,7]$ can provide a self-consistent first principle description of the turbulent structures but are much more computational demanding. The two approaches are complementary and both rely on the same set of fluid equations describing mass, momentum and energy balance of the various plasma species.

The most common set of equations is the Braginskii's equations [8] providing for the first time in the 1960's a collisional closure for the pressure tensor, friction forces and heat fluxes for a highly magnetized plasma. However, the collisional closure presented by Braginskii is derived for a simple electron-hydrogen plasma. In this contribution, we worked on the multi-species extension of Braginskii like equations proposed by Zhdanov [1]. This multicomponent closure based on Grad's method has already been used in the communinity $[9,10,11]$. It does not make assumptions on impurity concentration or mass ratio in between the different species. It is thus of particular interest to simulate reactor plasmas where a mix of Deuterium, Tritium and Helium ions takes place, the species having quite similar mass and concentration.

In this contribution we apply Zhdanov closure to the code SOLEDGE3X-EIRENE that implements a fluid model to describe the plasma either in 2D to make transport simulations or in 3D to run turbulent or 3D-transport simulations. In a first part, we present the multi-species SOLEDGE3X-EIRENE equations. In particular, a special attention is paid to describe the multi-species closure terms. To do so, Zhdanov closure is rewritten in a matricial form in order to relate the parallel heat fluxes $\left(q_{\alpha Z}\right)$ and parallel friction forces $\left(R_{\alpha Z}\right)$ felt by one component of species $\alpha$ at charge state component $Z$, because of parallel velocity $\left(w_{\beta \zeta}\right)$ and parallel temperature gradient $\left(\nabla T_{\beta \zeta}\right)$ of a another component of species $\beta$ at charge state $\zeta$. This relation takes the form:

$$
\left(\begin{array}{c}
q_{\alpha Z} \\
R_{\alpha Z}
\end{array}\right)=M_{\alpha Z, \beta \zeta} \cdot\left(\begin{array}{c}
\nabla T_{\beta \zeta} \\
w_{\beta \zeta}
\end{array}\right)
$$

The matrix $M$ contains in particular parallel heat conductivities as well as coefficient for thermal and drag forces.

Zhdanov closure is compared to Braginskii closure for a simple electron+hydrogen plasma. Also, in order to check the consistency of Zhdanov closure, a comparison is made between two simulations of a pure deuterium plasma: in the first one, deuterons are considered as a single species (standard treatment); in the second one the deuteron population is splitted into two different species. The total input power and particle input being the same, the two simulations should give exactly the same results if the coupling terms in between the two deuteron species are correct.

Finally, an example of application to a JET D-T fusion plasma is performed and reported in the last section. 


\section{Multi-component plasma SOLEDGE equations}

The SOLEDGE3X has been developed in the past few years to address plasma-wall interaction in tokamak plasmas taking into account the realistic wall geometry as much as possible. It merges the codes SOLEDGE2D and TOKAM3X previously developed by the same team. Thanks to immersed boundary condition methods [12], the code can simulate the plasma up to the first wall while using a magnetic field aligned grid. The physical model has been improved over the year to include neutrals via coupling the code to EIRENE [13], drifts [14] and impurities [9] since the code can now handle an arbitrary number of species. We denote by the subscript $\alpha Z$ the ion species of charged number $Z$ and of chemical element $\alpha$. The subscript $e$ is used for electrons.

\subsection{Mass balance}

For all ion species, the particle balance is given by:

$$
\partial_{t} n_{\alpha Z}+\vec{\nabla} \cdot\left(n_{\alpha Z} \vec{v}_{\alpha Z}\right)=S_{\alpha Z}^{n}
$$

where the velocity vector $\vec{v}_{\alpha Z}$ is decomposed as

$$
\vec{v}_{\alpha Z}=v_{\|, \alpha Z} \vec{b}+\vec{v}^{E}+\vec{v}_{\alpha Z}^{\star}+\vec{v}_{\alpha Z}^{p}+\vec{v}_{\alpha Z}^{a}
$$

where

- $v_{\|, \alpha Z}$ is the parallel velocity along the magnetic field unit vector $\vec{b}=\vec{B} / B$

- $\vec{v}^{E}=\frac{\vec{E} \times \vec{B}}{B^{2}}$ is the E cross B drift velocity

- $\vec{v}_{\alpha Z}^{\star}=\frac{\vec{B} \times \vec{\nabla} p_{\alpha Z}}{Z e n_{\alpha Z} B^{2}}$ is the diamagnetic drift velocity

- $\vec{v}_{\alpha Z}^{p}=-\frac{1}{n_{\alpha Z}}\left\{\partial_{t} \vec{\omega}_{\alpha Z}+\vec{\nabla} \cdot\left(\vec{v}_{\alpha Z}^{(0)} \otimes \vec{\omega}_{\alpha Z}\right)\right\}$ is the polarization drift with

$$
\vec{\omega}_{\alpha Z}=\frac{m_{\alpha}}{Z e B^{2}}\left(n_{\alpha Z} \vec{\nabla}_{\perp} \phi+\frac{1}{Z e} \vec{\nabla}_{\perp} p_{\alpha Z}\right)
$$

$\phi$ denoting the electric potential and where the first order velocity $\vec{v}_{\alpha Z}^{(0)}$ is the species velocity $\vec{v}_{\alpha Z}$ without the polarization drift.

- $\vec{v}_{\alpha Z}^{a}=-D_{\alpha Z} \frac{\vec{\nabla}_{\perp} n_{\alpha Z}}{n_{\alpha Z}}$ is the anomalous perpendicular velocity that is used to emulate the turbulent transport when the code is used as a transport code. The diffusion coefficient $D_{\alpha Z}$ takes value from empirical considerations or reduced turbulence models [15].

The volumic source term $S_{\alpha Z}^{n}$ describes particle sources and sinks due to ionization and recombination processes. It takes the form

$$
\begin{aligned}
S_{\alpha Z}^{n}= & -n_{e} n_{\alpha Z}\left(\langle\sigma v\rangle_{\mathrm{iz}, \alpha Z}+\langle\sigma v\rangle_{\mathrm{rec}, \alpha Z}\right) \\
& +n_{e} n_{\alpha Z-1}\langle\sigma v\rangle_{\mathrm{iz}, \alpha Z-1}+n_{e} n_{\alpha Z+1}\langle\sigma v\rangle_{\mathrm{rec}, \alpha Z+1}
\end{aligned}
$$

For ionization and recombination processes involving neutrals, the source term is computed by EIRENE. 
No transport equation is solved for electron density. It is computed from plasma quasineutrality assumption:

$$
n_{e}=\sum_{\alpha, Z} Z n_{\alpha Z}
$$

\subsection{Parallel momentum balance}

For each ion species, the following parallel momentum balance is solved:

$$
\begin{aligned}
\partial_{t}\left(m_{\alpha} n_{\alpha Z} v_{\|, \alpha Z}\right)+\vec{\nabla} \cdot\left(m_{\alpha} n_{\alpha Z} v_{\|, \alpha Z} \vec{v}_{\alpha Z}\right)= & -\nabla_{\|} p_{\alpha Z}+Z e n_{\alpha Z} E_{\|} \\
& +\vec{\nabla} \cdot\left(m_{\alpha} n_{\alpha Z} \nu_{\alpha Z} \vec{\nabla}_{\perp} v_{\|, \alpha Z}\right) \\
& +R_{\alpha Z}+S_{\alpha Z}^{v_{\|}}+\Sigma_{\alpha Z}^{v_{\|}}
\end{aligned}
$$

where $\nu_{\alpha Z}^{a}$ is the anomalous viscosity describing turbulent cross-field transport of parallel momentum in shear layers (set to classical value when the code is used in 3D to solve turbulence). The term $R_{\alpha Z}$ is the parallel friction force resulting from elastic collisions in between the different species. It can be decomposed as $R_{\alpha Z}=\sum_{\beta \zeta} R_{\alpha Z, \beta \zeta}$ where one exhibits the different contributions due to binary interactions between ion species $(\alpha, Z)$ and ion species $(\beta, \zeta)$. The elastic collision processes conserve parallel momentum giving $R_{\alpha Z, \beta \zeta}+R_{\beta \zeta, \alpha Z}=0$. Following Zhdanov closure, one can evaluate the friction forces from different species temperature gradients and parallel velocities. The detail of the closure is given in section 3. The volumic source term $S_{\alpha Z}^{v_{\|}}$accounts for momentum exchange in between species in the ionization-recombination processes. It is given by:

$$
\begin{aligned}
\frac{S_{\alpha Z}^{v_{\|}}}{m_{\alpha}}= & -n_{e} n_{\alpha Z} v_{\|, \alpha Z}\left(\langle\sigma v\rangle_{\mathrm{iz}, \alpha Z}+\langle\sigma v\rangle_{\mathrm{rec}, \alpha Z}\right) \\
& +n_{e} n_{\alpha Z-1} v_{\|, \alpha Z-1}\langle\sigma v\rangle_{\mathrm{iz}, \alpha Z-1}+n_{e} n_{\alpha Z+1} v_{\|, \alpha Z+1}\langle\sigma v\rangle_{\mathrm{rec}, \alpha Z+1}
\end{aligned}
$$

Finally, the source term $\Sigma_{\alpha Z}^{v_{\|}}$accounts for other momentum exchange processes such as charge exchange.

Parallel electron velocity is obtained from parallel current definition $j_{\|}=-e n_{e} v_{\|, e}+$

$\sum_{\alpha Z} Z n_{\alpha Z} v_{\|, \alpha Z}$ where the parallel current is obtained by solving current balance (see section 2.4. When the charge balance is not solved, one assumes $j_{\|}=0$ (ambipolarity) hence $v_{\|, e}=n_{e}^{-1} \sum_{\alpha Z} Z n_{\alpha Z} v_{\|, \alpha Z}$. We neglect electrons inertia which provides an expression for the parallel electric field from electron parallel momentum equation:

$$
0=-\nabla_{\|} p_{e}-e n_{e} E_{\|}+R_{e} \quad \text { or } \quad E_{\|}=-\frac{\nabla_{\|} p_{e}}{e n_{e}}+\frac{R_{e}}{e n_{e}}
$$

\subsection{Energy balance}

For all species (including electrons), the "total" energy balance is solved where the "total" energy is defined by

$$
\mathcal{E}_{\alpha Z}=\frac{3}{2} k_{B} n_{\alpha Z} T_{\alpha Z}+\frac{1}{2} m_{\alpha} n_{\alpha Z} v_{\|, \alpha Z}^{2}
$$

This quantity is not in fact the total energy since one could include in addition perpendicular kinetic energy and electrostatic potential energy. The consequence of 
not considering these terms on the conservative form of the equation will be discussed in the next paragraph. Moreover, in practice electron kinetic energy is neglected over electron internal energy. The "total" energy equation is given by

$$
\begin{aligned}
\partial_{t} \mathcal{E}_{\alpha Z}+\vec{\nabla} & \left(\mathcal{E}_{\alpha Z} \vec{v}_{\alpha Z}+p_{\alpha Z} v_{\|, \alpha Z} \vec{b}+q_{\alpha Z} \vec{b}\right)=Z e n_{\alpha Z} v_{\|, \alpha Z} E_{\|} \\
& +\vec{\nabla} \cdot\left(\frac{1}{2} m_{\alpha} n_{\alpha Z} \nu_{\alpha Z} \vec{\nabla}_{\perp} v_{\|, \alpha Z}\right)+\vec{\nabla} \cdot\left(k_{B} n_{\alpha Z} \chi_{\alpha Z} \vec{\nabla}_{\perp} T_{\alpha Z}\right) \\
& -p_{\alpha Z} \vec{\nabla} \cdot \vec{v}_{\perp, \alpha Z}+v_{\|, \alpha Z} R_{\alpha Z}+Q_{\alpha Z}+S_{\alpha Z}^{\mathcal{E}}+\Sigma_{\alpha Z}^{\mathcal{E}}
\end{aligned}
$$

where $\vec{v}_{\perp, \alpha Z}$ gathers all drifts velocities. The parallel heat flux is denoted $q_{\alpha Z}$ and also find an expression from Zhdanov closure that generalizes Spitzer-Härm heat conduction closure. In the RHS, one introduces an anomalous perpendicular heat conductivity $\chi_{\alpha Z}$ that takes value from empirical considerations (set to classical value when the code is used in $3 \mathrm{D}$ to solve turbulence). The term $Q_{\alpha Z}$ describes energy exchange in between species due to collisions and is also expressed in the next section about collisional closure. The volumic source term $S_{\alpha Z}^{\mathcal{E}}$ denotes energy source ans sinks due to ionization/recombination processes and takes the form:

$$
\begin{aligned}
S_{\alpha Z}^{\mathcal{E}}= & -n_{e} \mathcal{E}_{\alpha Z}\left(\langle\sigma v\rangle_{\mathrm{iz}, \alpha Z}+\langle\sigma v\rangle_{\mathrm{rec}, \alpha Z}\right) \\
& +n_{e} \mathcal{E}_{\alpha Z-1}\langle\sigma v\rangle_{\mathrm{iz}, \alpha Z-1}+n_{e} \mathcal{E}_{\alpha Z+1}\langle\sigma v\rangle_{\mathrm{rec}, \alpha Z+1}
\end{aligned}
$$

Finally, the source term $\Sigma_{\alpha Z}^{\mathcal{E}}$ denotes other volumic energy sources such as radiation losses (for electrons), charge exchange energy losses or external heating.

\subsection{Charge balance}

Assuming quasineutrality, the charge balance reduces to

$$
\vec{\nabla} \cdot \vec{j}=0
$$

The current is decomposed as

$$
\vec{j}=j_{\|} \vec{b}+\vec{j}^{\star}+\vec{j}^{p}
$$

where

- $j_{\|}$denotes the parallel current given by the generalized Ohm's law:

$$
j_{\|}=\sigma_{\|}\left(-\nabla_{\|} \phi-\frac{1}{e n_{e}}\left(\nabla_{\|} p_{e}-R_{e}^{T}\right)\right)
$$

$\sigma_{\|}$denoting the parallel electric conductivity and $R_{e}^{T}$ the thermal contribution of the friction force. The parallel conductivity is a collisional term that requires a closure. It is not discussed in this contribution.

- $\vec{j}^{\star}$ is the diamagnetic current given by $\vec{j}^{\star}=\sum_{e, \alpha, Z} Z e n_{\alpha Z} \vec{v}_{\alpha Z}^{\star}$

- $\vec{j}^{p}$ is the polarization current given by $\vec{j}^{p}=\sum_{\alpha, Z} Z e n_{\alpha Z} \vec{v}_{\alpha Z}^{p}$ 
Given the expression of the polarization velocity, one can show [14] that the current balance can be rewritten as a vorticity equation where the vorticity $\Omega$ is defined by $\Omega=\vec{\nabla} \cdot\left(\sum_{\alpha, Z} Z e \vec{\omega}_{\alpha Z}\right)$ where $\vec{\omega}_{\alpha Z}$ is given by Equation 4 . The vorticity equation takes the form:

$$
\partial_{t} \Omega+\vec{\nabla} \cdot \vec{F}_{\Omega}=\vec{\nabla} \cdot\left(j_{\|} \vec{b}+\vec{j}^{\star}+\zeta \vec{\nabla}_{\perp} \Omega\right)
$$

where the flux of vorticity $\vec{F}_{\Omega}$ is given by

$$
\vec{F}_{\Omega}=\sum_{\alpha, Z} Z e \vec{\nabla} \cdot\left(\vec{v}_{\alpha Z} \otimes \vec{\omega}_{\alpha Z}\right)
$$

A diffusion term is added in the vorticity Equation 16 to take into account anomalous current and for stability reason. The diffusivity $\zeta$ takes either anomalous or classical values depending whether the code is used as a transport code or as a turbulence code.

\section{Zhdanov closure}

The remarkable work of Zhdanov [1] consisted in extending the hydrogenic magnetized plasma collisional closure to multi-component plasmas in the linear transport regime under weak-field conditions. It provides in particular expressions for the above mentioned friction forces $R_{\alpha Z}$, heat fluxes $q_{\alpha Z}$, energy coupling terms $Q_{\alpha Z}$ and plasma conductivity $\sigma_{\|}$. It does not make assumptions of trace impurities and all species are treated on the same footing. However, it does not provide explicit formulation for each of these terms. The species being intimately coupled between each other, the closure takes the form of a linear system of equations linking the different species plasma fields (in particular diffusion velocities and temperature gradients) to the quantities we want to express, that is in particular friction forces and heat fluxes.

\subsection{Friction forces and heat fluxes}

First of all, most of the Zhdanov closure algebra is performed in the frame of the local center of the mass, the velocity of which is given by

$$
v_{\|}=\frac{\sum_{\alpha Z} m_{\alpha} n_{\alpha Z} v_{\|, \alpha Z}}{\sum_{\alpha Z} m_{\alpha} n_{\alpha Z}}
$$

In this frame, the peculiar velocity of the component is denoted $w_{\alpha Z}=v_{\|, \alpha Z}-v_{\|}$. The purpose of this section is to express the friction force $R_{\alpha Z}$ and the heat flux $q_{\alpha} \ddagger$ as a linear combination of the different species parallel velocities and parallel temperature gradients as already expressed by the matrix product presented in the introduction in Equation 49. Several steps are required to express the matrix $M_{\alpha Z, \beta \zeta}$. First, physical

$\ddagger$ Careful: In Zhdanov book [1], the heat flux for each species is denoted $h_{\alpha Z}$. The notation $q_{\alpha Z}$ in [1] refers to the heat flux in the centre of mass frame (see Equation 2.2.4 in [1]), that is $\frac{5}{2} p_{\alpha Z} v_{\|}+q_{\alpha Z}=\frac{5}{2} p_{\alpha Z} v_{\|, \alpha Z}+h_{\alpha Z}$, hence $h_{\alpha Z}=q_{\alpha Z}-\frac{5}{2} p_{\alpha Z} w_{\alpha Z}$ (Equation 4.2 .4 in [1]). In this paper, we do not use the heat flux in the centre of mass frame and thus use the more common notation $q_{\alpha} Z$ for the heat flux instead of $h_{\alpha Z}$. 
quantites are defined for each chemical element, averaging over the different ionization states:

- density: $\bar{n}_{\alpha}=\sum_{Z} n_{\alpha Z}$

- effective $k^{t h}$ power of charge $(\forall k \in \mathbb{N}): \overline{Z_{\alpha}^{k}}=\bar{n}_{\alpha}^{-1} \sum_{Z} n_{\alpha Z} Z^{k}$

- velocity: $\bar{w}_{\alpha}=\sum_{Z} I_{\alpha Z} w_{\alpha Z}$ where $I_{\alpha Z}=\frac{n_{\alpha Z} Z^{2}}{\bar{n}_{\alpha} \bar{Z}_{\alpha}^{2}}$

- temperature gradient: $\overline{\nabla T}_{\alpha}=\left(\bar{n}_{\alpha} \overline{Z_{\alpha}^{1}}\right)^{-1} \sum_{Z} Z n_{\alpha Z} \nabla T_{\alpha Z}$

- pressure : $\bar{p}_{\alpha}=\sum_{Z} k_{B} n_{\alpha Z} T_{\alpha Z}$

In particular, expressions for velocity and temperature can be re-written in the matrix form:

$$
\left(\begin{array}{c}
\overline{\nabla T}_{\alpha} \\
\bar{w}_{\alpha}
\end{array}\right)=M 1 \cdot\left(\begin{array}{c}
\nabla T_{\beta Z} \\
w_{\beta Z}
\end{array}\right)=\left(\begin{array}{cc}
M 11 & 0 \\
0 & M 12
\end{array}\right) \cdot\left(\begin{array}{c}
\nabla T_{\beta Z} \\
w_{\beta Z}
\end{array}\right)
$$

where $M 11$ and $M 12$ are $N_{\text {chem }} \times N_{\text {spec }}$ rectangular matrices $\left(N_{\text {chem }}\right.$ denoting the number of chemical elements and $N_{\text {spec }}$ the number of species considering all ionization states). More precisely:

$$
M 11_{\alpha, \beta Z}=\frac{n_{\beta Z} Z}{\bar{n}_{\beta}} \delta_{\alpha \beta}
$$

and

$$
M 12_{\alpha, \beta Z}=I_{\beta Z} \delta_{\alpha \beta}
$$

From the averaged velocities and parallel gradients are computed averaged heat fluxes and averaged fifth order rank-1 fluid moments denoted $\bar{r}_{\alpha}$ from which the friction forces will be computed later. The following set of reduced equations is the keystone of Zhdanov closure:

$$
\begin{aligned}
\frac{5}{2} \bar{n}_{\alpha} k_{B} \overline{\nabla T}_{\alpha}=\sum_{\beta}\left[\frac{5}{2} \frac{\mu_{\alpha \beta}}{m_{\alpha}} \bar{G}_{\alpha \beta}^{(2)}\left(\bar{w}_{\alpha}-\bar{w}_{\beta}\right)+\bar{G}_{\alpha \beta}^{(5)} \frac{\bar{q}_{\alpha}}{p_{\alpha}}\right. \\
\left.+\bar{G}_{\alpha \beta}^{(6)} \frac{\bar{q}_{\beta}}{p_{\beta}}+\frac{\mu_{\alpha \beta}}{k_{B} T}\left(\bar{G}_{\alpha \beta}^{(9)} \frac{\bar{r}_{\alpha}}{p_{\alpha}}+\bar{G}_{\alpha \beta}^{(10)} \frac{\bar{r}_{\beta}}{p_{\beta}}\right)\right] \\
0=\sum_{\beta}\left[\frac{35}{2}\left(\frac{\mu_{\alpha \beta}}{m_{\alpha}}\right)^{2} \bar{G}_{\alpha \beta}^{(8)}\left(\bar{w}_{\alpha}-\bar{w}_{\beta}\right)+7 \frac{\mu_{\alpha \beta}}{m_{\alpha}}\left(\bar{G}_{\alpha \beta}^{(9)} \frac{\bar{q}_{\alpha}}{p_{\alpha}}\right.\right. \\
\left.\left.+\bar{G}_{\alpha \beta}^{(10)} \frac{\bar{q}_{\beta}}{p_{\beta}}\right)+\frac{m_{\alpha}}{k_{B} T} \bar{G}_{\alpha \beta}^{(11)} \frac{\bar{r}_{\alpha}}{p_{\alpha}}+\frac{m_{\beta}}{k_{B} T} \bar{G}_{\alpha \beta}^{(12)} \frac{\bar{r}_{\beta}}{p_{\beta}}\right]
\end{aligned}
$$

It can be re-written in the matrix form as:

$$
M 2 \cdot\left(\begin{array}{c}
\overline{\nabla T}_{\beta} \\
\bar{w}_{\beta}
\end{array}\right)=M 3 \cdot\left(\begin{array}{c}
\bar{q}_{\beta} \\
\bar{r}_{\beta}
\end{array}\right)
$$

where $M 2$ is a square $2 N_{\text {chem }} \times 2 N_{\text {chem }}$ matrix where the coefficients are given for all $[\alpha, \beta] \in\left[1, N_{\text {chem }}\right] \times\left[1, N_{\text {chem }}\right]:$

- $M 2_{\alpha, \beta}=\frac{5}{2} \bar{n}_{\alpha} k_{B} \delta_{\alpha \beta}$ 
- $M 2_{\alpha+N_{\text {chem }, \beta}=0}$

- $M 2_{\alpha, \beta+N_{\text {chem }}}=-\frac{5}{2}\left(\sum_{\beta} \frac{\mu_{\alpha \beta}}{m_{\alpha}} \bar{G}_{\alpha \beta}^{(2)}\right) \delta_{\alpha \beta}+\frac{5}{2} \frac{\mu_{\alpha \beta}}{m_{\alpha}} \bar{G}_{\alpha \beta}^{(2)}$

- $M 2_{\alpha+N_{\text {chem }}, \beta+N_{\text {chem }}}=-\frac{35}{2}\left(\sum_{\beta}\left(\frac{\mu_{\alpha \beta}}{m_{\alpha}}\right)^{2} \bar{G}_{\alpha \beta}^{(8)}\right) \delta_{\alpha \beta}+\frac{35}{2}\left(\frac{\mu_{\alpha \beta}}{m_{\alpha}}\right)^{2} \bar{G}_{\alpha \beta}^{(8)}$

The matrix $M 3$ is also a square $2 N_{\text {chem }} \times 2 N_{\text {chem }}$ matrix where the coefficients are given for all $[\alpha, \beta] \in\left[1, N_{\text {chem }}\right] \times\left[1, N_{\text {chem }}\right]$ :

- $M 3_{\alpha, \beta}=\left(\sum_{\beta} \frac{\bar{G}_{\alpha \beta}^{(5)}}{p_{\alpha}}\right) \delta_{\alpha \beta}+\frac{\bar{G}_{\alpha \beta}^{(6)}}{p_{\beta}}$

- $M 3_{\alpha+N_{\text {chem }}, \beta}=\left(\sum_{\beta} 7 \frac{\mu_{\alpha \beta}}{m_{\alpha}} \frac{\bar{G}_{\alpha \beta}^{(9)}}{p_{\alpha}}\right) \delta_{\alpha \beta}+7 \frac{\mu_{\alpha \beta}}{m_{\alpha}} \frac{\bar{G}_{\alpha \beta}^{(10)}}{p_{\beta}}$

- $M 3_{\alpha, \beta+N_{\text {chem }}}=\left(\sum_{\beta} \frac{\mu_{\alpha \beta}}{k_{B} T} \frac{\bar{G}_{\alpha \beta}^{(9)}}{p_{\alpha}}\right) \delta_{\alpha \beta}+\frac{\mu_{\alpha \beta}}{k_{B} T} \frac{\bar{G}_{\alpha \beta}^{(10)}}{p_{\beta}}$

- $M 3_{\alpha+N_{\text {chem }}, \beta+N_{\text {chem }}}=\left(\sum_{\beta} \frac{m_{\alpha}}{k_{B} T} \frac{\bar{G}_{\alpha \beta}^{(11)}}{p_{\alpha}}\right) \delta_{\alpha \beta}+\frac{m_{\beta}}{k_{B} T} \frac{\bar{G}_{\alpha \beta}^{(12)}}{p_{\beta}}$

A few notations are introduced above. First, the plasma common temperature $T$ is defined as

$$
T=\frac{\sum_{\alpha Z} n_{\alpha Z} T_{\alpha Z}}{\sum_{\alpha Z} n_{\alpha Z}}
$$

Secondly, the reduced mass $\mu_{\alpha \beta}=m_{\alpha} m_{\beta} /\left(m_{\alpha}+m_{\beta}\right)$. Then, the coefficients $\bar{G}_{\alpha \beta}^{(n)}$, combinations of collision times and species masses, are given in Appendix A.

The heat flux $q_{\alpha Z}$ for each ionization state can be computed from $\bar{q}_{\alpha}$ using Equation 26:

$$
\frac{q_{\alpha Z}}{p_{\alpha Z}}-\frac{\bar{q}_{\alpha}}{\bar{p}_{\alpha}}=\bar{n}_{\alpha} \tau_{\alpha} \tau_{\alpha \alpha}^{-1} c_{\alpha}^{(5)}\left(\frac{\overline{Z_{\alpha}^{2}}}{Z^{2}} \nabla T_{\alpha Z}-\overline{\nabla T} \bar{T}_{\alpha}\right)+c_{\alpha}^{(6)}\left(w_{\alpha Z}-\bar{w}_{\alpha}\right)
$$

The same kind of equation provides an expression for the fourth order moment $r_{\alpha Z}$ :

$$
\begin{aligned}
\frac{r_{\alpha Z}}{p_{\alpha Z}}-\frac{\bar{r}_{\alpha}}{\bar{p}_{\alpha}}= & \frac{k_{B} T}{m_{\alpha} S_{\alpha}^{(11)}}\left[-7 S_{\alpha}^{(9)} \bar{n}_{\alpha} \tau_{\alpha} \tau_{\alpha \alpha}^{-1} c_{\alpha}^{(5)}\left(\frac{\overline{Z_{\alpha}^{2}}}{Z^{2}} \nabla T_{\alpha Z}-\overline{\nabla T} T_{\alpha}\right)\right. \\
& \left.-\left(S_{\alpha}^{(8)}+7 S_{\alpha}^{(9)} c_{\alpha}^{(6)}\right)\left(w_{\alpha Z}-\bar{w}_{\alpha}\right)\right]
\end{aligned}
$$

where the collision times, the coefficients $S_{\alpha}^{(n)}$ and $c_{\alpha}^{(n)}$ are given in Appendix A. Once again, the above two equations can be re-written in the matrix form

$$
\left(\begin{array}{c}
q_{\alpha Z} \\
r_{\alpha Z}
\end{array}\right)=M 4 \cdot\left(\begin{array}{c}
\bar{q}_{\beta} \\
\bar{r}_{\beta}
\end{array}\right)+M 5 \cdot\left(\begin{array}{c}
\overline{\nabla T}_{\beta} \\
\bar{w}_{\beta}
\end{array}\right)+M 6 \cdot\left(\begin{array}{c}
\nabla T_{\beta \zeta} \\
w_{\beta \zeta}
\end{array}\right)
$$

where

- $M 4$ is a $2 N_{\text {spec }} \times 2 N_{\text {chem }}$ rectangular matrix given by

$$
M 4=\left(\begin{array}{cc}
M 41 & 0 \\
0 & M 41
\end{array}\right)
$$

where $\forall[\alpha Z, \beta] \in N_{\text {spec }} \times N_{\text {chem }}$ one has $M 41_{\alpha Z, \beta}=p_{\alpha Z} / p_{\beta} \delta_{\alpha \beta}$. 
- $M 5$ is a $2 N_{\text {spec }} \times 2 N_{\text {chem }}$ rectangular matrix given by

$$
M 5=\left(\begin{array}{ll}
M 51 & M 52 \\
M 53 & M 54
\end{array}\right)
$$

where $\forall[\alpha Z, \beta] \in N_{\text {spec }} \times N_{\text {chem }}$ one has

$$
\begin{aligned}
M 51_{\alpha Z, \beta} & =-p_{\alpha Z} \bar{n}_{\alpha} \tau_{\alpha} \tau_{\alpha \alpha}^{-1} c_{\alpha}^{(5)} \delta_{\alpha \beta} \\
M 52_{\alpha Z, \beta} & =-p_{\alpha Z} c_{\alpha}^{(6)} \delta_{\alpha \beta} \\
M 53_{\alpha Z, \beta} & =\frac{k_{B} T}{m_{\alpha} S_{\alpha}^{(11)}} 7 S_{\alpha}^{(9)} p_{\alpha Z} \bar{n}_{\alpha} \tau_{\alpha} \tau_{\alpha \alpha}^{-1} c_{\alpha}^{(5)} \delta_{\alpha \beta} \\
M 54_{\alpha Z, \beta} & =\frac{k_{B} T}{m_{\alpha} S_{\alpha}^{(11)}} p_{\alpha Z}\left(S_{\alpha}^{(8)}+7 S_{\alpha}^{(9)} c_{\alpha}^{(6)}\right) \delta_{\alpha \beta}
\end{aligned}
$$

- M6 is a $2 N_{\text {spec }} \times 2 N_{\text {spec }}$ square matrix given by

$$
M 6=\left(\begin{array}{ll}
M 61 & M 62 \\
M 63 & M 64
\end{array}\right)
$$

where $\forall[\alpha Z, \beta \zeta] \in N_{\text {spec }} \times N_{\text {spec }}$ one has

$$
\begin{aligned}
M 61_{\alpha Z, \beta \zeta} & =p_{\alpha Z} \bar{n}_{\alpha} \tau_{\alpha} \tau_{\alpha \alpha}^{-1} c_{\alpha}^{(5)} \frac{\overline{Z_{\alpha}^{2}}}{Z^{2}} \delta_{\alpha Z \beta \zeta} \\
M 62_{\alpha Z, \beta \zeta} & =p_{\alpha Z} c_{\alpha}^{(6)} \delta_{\alpha Z \beta \zeta} \\
M 63_{\alpha Z, \beta \zeta} & =-\frac{k_{B} T}{m_{\alpha} S_{\alpha}^{(11)}} 7 S_{\alpha}^{(9)} p_{\alpha Z} \bar{n}_{\alpha} \tau_{\alpha} \tau_{\alpha \alpha}^{-1} c_{\alpha}^{(5)} \frac{\overline{Z_{\alpha}^{2}}}{Z^{2}} \delta_{\alpha Z \beta \zeta} \\
M 64_{\alpha Z, \beta \zeta} & =-\frac{k_{B} T}{m_{\alpha} S_{\alpha}^{(11)}} p_{\alpha Z}\left(S_{\alpha}^{(8)}+7 S_{\alpha}^{(9)} c_{\alpha}^{(6)}\right) \delta_{\alpha Z \beta \zeta}
\end{aligned}
$$

At last, the friction force $R_{\alpha Z}$ is given by Equation (8.1.3) in [1]:

$$
\begin{aligned}
R_{\alpha Z}= & \sum_{\beta, \zeta}\left[\bar{G}_{\alpha Z \beta \zeta}^{(1)}\left(w_{\alpha Z}-w_{\beta \zeta}\right)+\frac{\mu_{\alpha \beta}}{k_{B} T} \bar{G}_{\alpha Z \beta \zeta}^{(2)}\left(\frac{q_{\alpha Z}}{m_{\alpha} n_{\alpha Z}}-\frac{q_{\beta \zeta}}{m_{\beta} n_{\beta \zeta}}\right)\right. \\
& \left.+\left(\frac{\mu_{\alpha \beta}}{k_{B} T}\right)^{2} \bar{G}_{\alpha Z \beta \zeta}^{(8)}\left(\frac{r_{\alpha Z}}{m_{\alpha} n_{\alpha Z}}-\frac{r_{\beta \zeta}}{m_{\beta} n_{\beta \zeta}}\right)\right]
\end{aligned}
$$

giving in the matrix formulation:

$$
\left(\begin{array}{c}
q_{\alpha Z} \\
R_{\alpha Z}
\end{array}\right)=M 7 \cdot\left(\begin{array}{c}
\nabla T_{\beta \zeta} \\
w_{\beta \zeta}
\end{array}\right)+M 8 \cdot\left(\begin{array}{c}
q_{\beta \zeta} \\
r_{\beta \zeta}
\end{array}\right)
$$

where

- $M 7$ is a $2 N_{\text {spec }} \times 2 N_{\text {spec }}$ square matrix given by

$$
M 7=\left(\begin{array}{cc}
0 & 0 \\
0 & M 71
\end{array}\right)
$$

with $\forall[\alpha Z, \beta \zeta] \in N_{\text {spec }} \times N_{\text {spec }}$ :

$$
M 71_{\alpha Z, \beta \zeta}=-\bar{G}_{\alpha Z \beta \zeta}^{(1)}+\left(\sum_{\beta, \zeta} \bar{G}_{\alpha Z \beta \zeta}^{(1)}\right) \delta_{\alpha Z \beta \zeta}
$$


- $M 8$ is a $2 N_{\text {spec }} \times 2 N_{\text {spec }}$ square matrix given by

$$
M 8=\left(\begin{array}{cc}
\mathbb{I} & 0 \\
M 81 & M 82
\end{array}\right)
$$

with $\forall[\alpha Z, \beta \zeta] \in N_{\text {spec }} \times N_{\text {spec }}$ :

$$
\begin{aligned}
M 81_{\alpha Z, \beta \zeta} & =-\frac{\mu_{\alpha \beta}}{k_{B} T} \frac{\bar{G}_{\alpha Z \beta \zeta}^{(2)}}{m_{\beta} n_{\beta \zeta}}+\left(\sum_{\beta, \zeta} \frac{\mu_{\alpha \beta}}{k_{B} T} \frac{\bar{G}_{\alpha Z \beta \zeta}^{(2)}}{m_{\alpha} n_{\alpha Z}}\right) \delta_{\alpha Z \beta \zeta} \\
M 82_{\alpha Z, \beta \zeta} & =-\left(\frac{\mu_{\alpha \beta}}{k_{B} T}\right)^{2} \frac{\bar{G}_{\alpha Z \beta \zeta}^{(8)}}{m_{\beta} n_{\beta \zeta}}+\left(\sum_{\beta, \zeta}\left(\frac{\mu_{\alpha \beta}}{k_{B} T}\right)^{2} \frac{\bar{G}_{\alpha Z \beta \zeta}^{(8)}}{m_{\alpha} n_{\alpha Z}}\right) \delta_{\alpha Z \beta \zeta}(46)
\end{aligned}
$$

Once all the above matrices calculated, that is the eight matrices $M 1 \ldots M 8$, the final matrix linking heat fluxes and friction forces to temperature gradients and velocities can be calculated. From Equation 24, one finds

$$
\left(\begin{array}{c}
\bar{q}_{\beta} \\
\bar{r}_{\beta}
\end{array}\right)=M 3^{-1} \cdot M 2 \cdot\left(\begin{array}{c}
\overline{\nabla T}_{\beta} \\
\bar{w}_{\beta}
\end{array}\right)
$$

Reporting in Equation 28 gives

$$
\left(\begin{array}{c}
q_{\alpha Z} \\
r_{\alpha Z}
\end{array}\right)=\left[M 4 \cdot M 3^{-1} \cdot M 2+M 5\right] \cdot\left(\begin{array}{c}
\overline{\nabla T}_{\beta} \\
\bar{w}_{\beta}
\end{array}\right)+M 6 \cdot\left(\begin{array}{c}
\nabla T_{\beta \zeta} \\
w_{\beta \zeta}
\end{array}\right)
$$

Combining with Equation 19 and 41 finally gives the desired expression

$$
\left(\begin{array}{c}
q_{\alpha Z} \\
R_{\alpha Z}
\end{array}\right)=M_{\alpha Z, \beta \zeta} \cdot\left(\begin{array}{c}
\nabla T_{\beta \zeta} \\
w_{\beta \zeta}
\end{array}\right)
$$

where the matrix $M$ is given by

$$
M=M 7+M 8 \cdot\left[\left(M 4 \cdot M 3^{-1} \cdot M 2+M 5\right) \cdot M 1+M 6\right]
$$

Once each individual matrix computed, the final matrix $M$ is quite straightforward to obtain by matrix product. The only matrix inversion required implies the matrix $M 3$ which is a relatively small $2 N_{\text {chem }} \times 2 N_{\text {chem }}$ matrix. The direct numerical inversion can be done easily with a linear solver such as LAPACK. Most of the numerical cost in the matrix approach relies in fact in computing the different collision times and Zhdanov's collision coefficients $\bar{G}_{\alpha \beta}^{(n)}, S_{\alpha}^{(n)} \ldots$

\section{Boundary conditions}

SOLEDGE3X being based on a finite volume numerical scheme, setting the boundary conditions consists in setting particle, momentum and energy fluxes on faces at the boundary of the domain, in particular on the wall. 


\subsection{Particle fluxes on the wall}

Particle fluxes on the wall are given by the Bohm-Chodura boundary condition: the particle flux on the wall is set to be greater or equal to the particle flux that would result from a plasma flowing at sound speed in the direction parallel to the magnetic field. This condition guarantees in particular that whatever the value and the orientation of the drift velocities, the flux on the wall will always be a net outflux of particle. This boundary condition can be summarized as

$$
\left|\vec{\phi}_{n} \cdot \vec{n}_{\text {wall }}\right| \geq\left|n c_{s} \vec{b} \cdot \vec{n}_{\text {wall }}\right|
$$

where $\vec{\phi}_{n}$ denotes the particle flux. In the present implementation, every ion species follows its own sound speed on the wall. For a collisional multi-component plasma, other expression for the boundary condition exist in the litterature, in particular using a common sound speed followed by all ion species on the wall [16]. These boundary conditions will be considered for implementation in the future. In addition, one forces $\nabla_{\|} n=0$ to set density in ghost cells on the wall.

\subsection{Momentum fluxes on the wall}

On the flux of momentum, one sets Neumann boundary conditions on parallel momentum $\nabla_{\|} v_{\|}=0$. The flux is not forced, one let the free streaming advected flux on the wall.

\subsection{Energy fluxes on the wall}

The energy flux is set according to simplified sheath properties that link energy and particle fluxes on the wall. The sheath transmission factor $\gamma$ estimates the average energy of the particles crossing the sheath electrostatic barrier. The total energy flux on the wall is thus given by:

$$
\vec{\phi}_{E \alpha, s e}=\left(\gamma_{\alpha} T_{\alpha}+\frac{1}{2} m_{\alpha} v_{\|, \alpha}^{2}\right) \vec{\phi}_{n \alpha, s e}
$$

A typical value for electron sheath tranmission factor is $\gamma_{e}=4.5$ providing that only the fast electron in the tail of the distribution can cross the sheath barrier. For ions, a typical value for the sheath transmission factor is $\gamma_{i}=2.5$ which is reminiscent from the free streaming advected flux, the sheath letting all ions go through. Other values can be found in the litterature [17] and improved sheath models should be implemented in the future.

\subsection{Current on the wall}

In a similar fashion as the energy flux, the current on the wall can be set proportionaly to the particle flux. The following expression is used:

$$
\vec{j}_{\text {wall }}=\vec{j}_{\text {sat }}\left[1-\exp \left(1-\frac{\phi}{\Lambda T_{e}}\right)\right]
$$


where the saturation current is given by ion saturation current $\vec{j}_{s a t}=\sum_{\alpha, Z} Z e \vec{\phi}_{n \alpha Z}$.

\section{Verification of the implementation}

\subsection{Verification of the operators}

SOLEDGE3X is based on a finite-volume, flux surface aligned, multi-domain algorithm [18]. In order to verify the implementation of the different operators, the method of manufactured solution (MMS) is used [19]. It consists in comparing numerical solution with an analytical solution. The method can be summarized as follow. Let us consider an operator $\mathcal{O}$ to test (for instance advection, diffusion...). We consider the equation:

$$
\partial_{t} X+\mathcal{O}(X)=S
$$

A priori, the analytical steady-state solution $\tilde{X}$ associate to the arbitrary source $S$ of the system is not obvious since it requires inverting the operator $\tilde{X}=\mathcal{O}^{-1}(S)$. The MMS propose simply to choose an analytical solution $\tilde{X}_{M M S}$ and compute to associated source by a direct analytical computation $S_{M M S}=\mathcal{O}^{\text {analyt. }}\left(\tilde{X}_{M M S}\right)$. One thus solves numerically the system:

$$
\partial_{t} X+\mathcal{O}(X)=S_{M M S} \equiv \mathcal{O}^{\text {analyt. }}\left(\tilde{X}_{M M S}\right)
$$

which by construction admit $\tilde{X}=\tilde{X}_{M M S}$ as a steady-state solution. In practice, we choose a circular cross-section toroidal domain. We impose a steady-state analytical solution for density, temperature and velocity fields based on trigonometric fonctions. One initiates the plasma to the analytical steady-state solution and perform one time step $\delta t$. The numerical departure from steady-state is measured and characterize the error $\varepsilon=\left\|X-\tilde{X}_{M M S}\right\| / \delta t$. The numerical test is performed for different grid resolutions. The error is expected to decrease accordingly to the order of the numerical scheme. The MMS can be used with a regular grid (see Figure 1) or irregular grid to test the ability of the code to deal with non-constant spatial discretization.
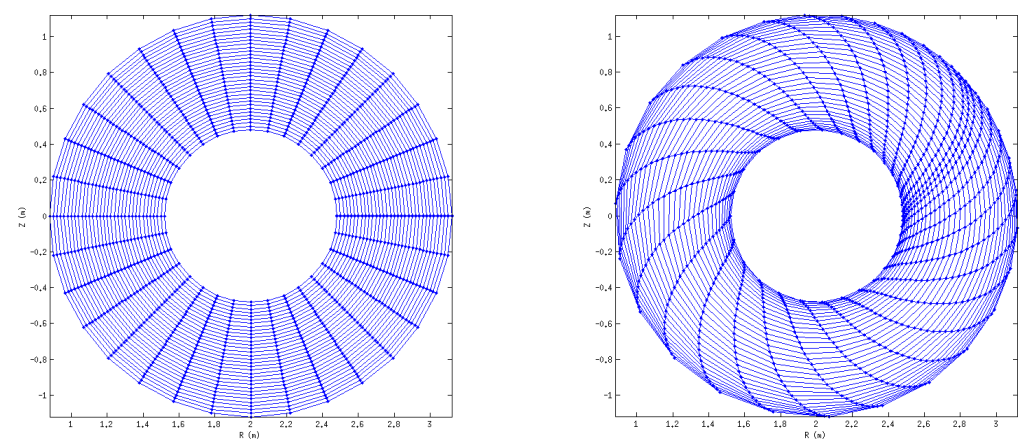

Figure 1. Description of regular and irregular grid used for meshing the circular cross-section of the domain used for the MMS

The different operators are tested: 

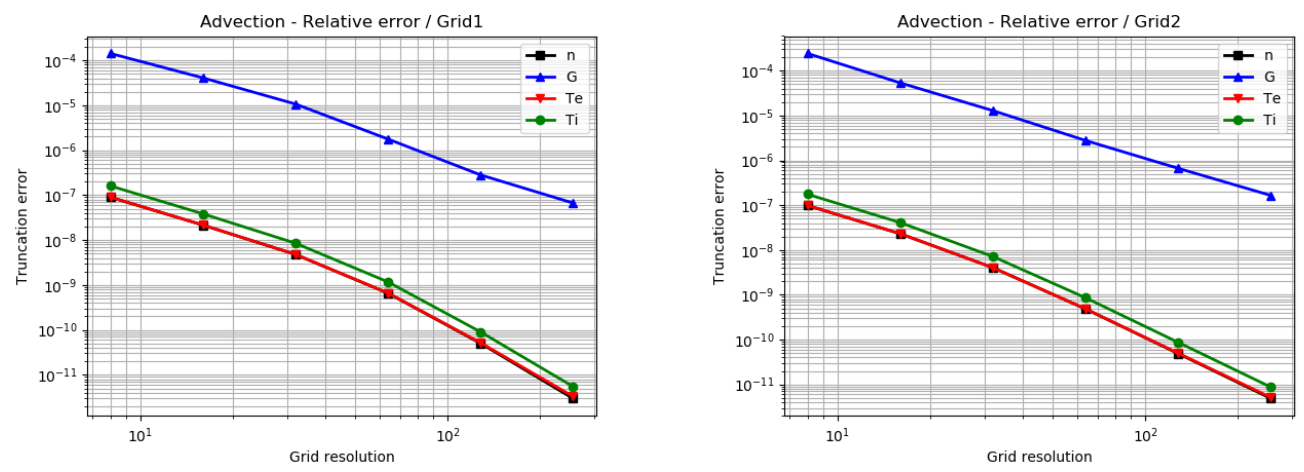

Figure 2. Relative error as a function of grid resolution for advection operators. Left: regular grid, Right: irregular grid.

- Parallel advection (Figure 2):

One finds a spatial discretization order between 2 and 3.

- Parallel diffusion (Figure 3):
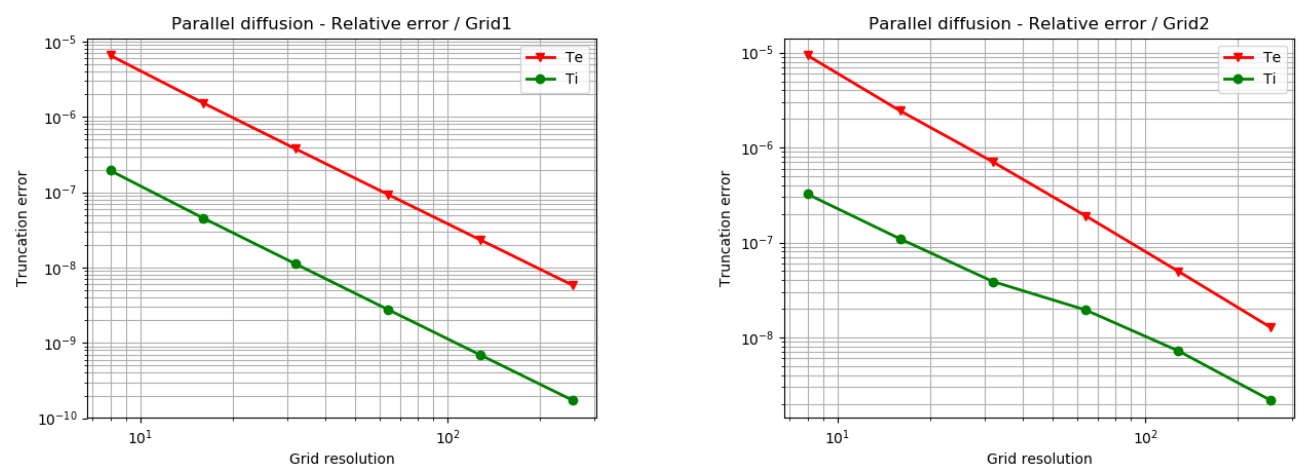

Figure 3. Relative error as a function of grid resolution for parallel diffusion operators. Left: regular grid, Right: irregular grid.

One finds a spatial discretization order of 2.

- Perpendicular diffusion (Figure 4):

One finds a spatial discretization order of 2 .

\subsection{Verification of the closure}

Veryfing Zhdanov closure is not as simple as the verification of the operators described in the previous paragraph. Indeed, a method of manufactured solution appear quite cumbersome since Zhdanov closure does not provide an explicit formulation for collisional terms. We propose here a "weak verification" of Zhdanov closure implementation (and behavior). In order to verify the multi-species collisional closure, a simple numerical test case is proposed. The simulation set-up consists in simulating a pure Deuterium plasma in a circular limited configuration following three approaches (in the following, if not explicitly written, all quantities are given in the MKS convention): 

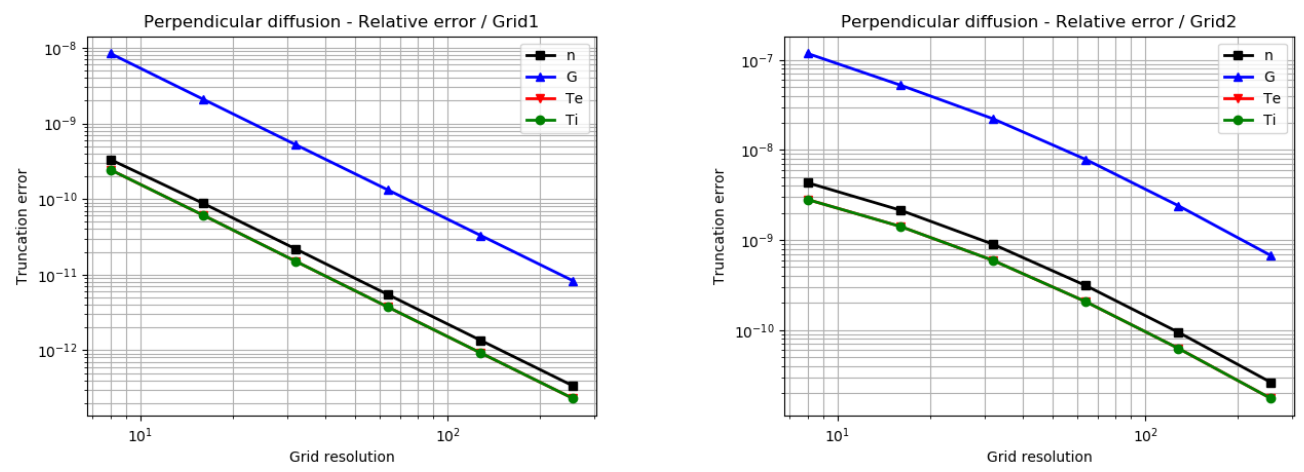

Figure 4. Relative error as a function of grid resolution for perpendicular diffusion operators. Left: regular grid, Right: irregular grid.

(i) As a reference case, one simulates a two fluids plasma of deuterons and electrons where standard Braginskii closure is used, that is:

- For the friction force:

$$
R_{e}=-R_{i}=-m_{e} n_{e}\left(v_{\|, e}-v_{\|, i}\right) \nu_{e i}^{m o m}-0.71 n_{e} \nabla_{\|} T_{e}
$$

where

$$
\nu_{e i}^{m o m}=1.45 \times 10^{-12} \ln \Lambda n_{e} T_{e}^{-3 / 2} \quad\left[s^{-1}\right]
$$

where $n_{e}$ is expressed in $m^{-3}$ and $T_{e}$ in $e V$.

- For the heat fluxes: $q_{e}=-\kappa_{e}^{0} T_{e}^{5 / 2} \nabla_{\|} T_{e}$ and $q_{i}=-\kappa_{D^{+}}^{0} T_{D^{+}}^{5 / 2} \nabla_{\|} T_{D^{+}}$where the coefficient $\kappa_{e}^{0}$ is given by

$$
\kappa_{e}^{0}=\frac{30692}{\ln \Lambda} \approx 2000 \quad\left[\mathrm{Wm}^{-1} \mathrm{eV}^{-7 / 2}\right]
$$

and

$$
\kappa_{D^{+}}^{0}=\frac{1249}{m_{i}^{1 / 2} \ln \Lambda} \approx 60 \quad\left[\mathrm{Wm}^{-1} \mathrm{eV}^{-7 / 2}\right]
$$

where $m_{i}$ is expressed in amu and where the Coulomb logarithm is given by the expression A.18 in the appendice. The expressions above are taken from [17].

(ii) A two fluids plasma of deuterons and electrons is simulated with Zhdanov closure. The purpose here is to see if the collisions between ions and electrons are well treated by Zhdanov closure

(iii) A three fluids plasma of deuterons+deuterons+electrons where the deuterium ion population is splitted into two different fluids. The purpose is to test Zhdanov collisional closure between the two populations of deuterium.

The three cases are simulated on the same circular mesh plotted in Figure 5. The simulation domain extends from the core flux surface located at $r / a=0.8$ up to the wall. On the core boundary, Dirichlet boundary conditions are used for temperature (namely $T_{e}=T_{D^{+}}=50 \mathrm{eV}$ ) as well as for density $n_{e}=n_{D^{+}}=2 \cdot 10^{19} \mathrm{~m}^{-3}$. For the third case where deuteron population is splitted into two fluids, one imposes 
$n_{D^{+}}=1.2 \cdot 10^{19} \mathrm{~m}^{-3}$ for the first one, $n_{D^{+}}=0.8 \cdot 10^{19} \mathrm{~m}^{-3}$ for the second one in order to have the same total deuterium density. Homogeneous anomalous diffusivities are considered: $D_{D^{+}}=1 \mathrm{~m}^{2} \mathrm{~s}^{-1}, \nu_{D^{+}}=1 \mathrm{~m}^{2} \mathrm{~s}^{-1}$ and $\chi_{e}=\chi_{D^{+}}=2 \mathrm{~m}^{2} \mathrm{~s}^{-1}$. No parallel flux limiters are considered. EIRENE is not used in this set of simulations so sources due to neutrals are set to zero (no recycling). Figure 5 shows radial profiles of density and temperature for ions and electrons at the outboard midplane. A very good agreement is found between the three simulations: a maximum relative error of $1.4 \%$ is found comparing the different density and temperature profiles (the error being defined as $\left.\varepsilon=\left\|\frac{X_{1}-X_{2}}{X_{1}}\right\|_{\infty}\right)$. An almost perfect match between electron density profiles show the good overall agreement while ion density profiles show the fractionning of deuterium density over the two fluids for the third case (iii). Concerning temperature, the four deuterium temperature profiles collapses with each other. In particular, when deuterium is splitted in two, the two fluids are found to have the same temperature (as could be expected for any intensive physical quantity).

In the case where deuterium is splitted in two, it is interesting to notice that the heat flux of a type of deuterium is given by a combination of the temperature gradients of the two types of deuterium. In that sense, we notice that the matrix linking heat fluxes and temperature gradient is not diagonal (see Equation B.4 in Appendix Appendix A). Here is a example of decomposition:

$$
\begin{aligned}
q_{D^{+}(1)}= & -30 T_{D^{+}(1)}^{5 / 2} \nabla_{\|} T_{D^{+}(1)} \\
& -4.34 T_{D^{+}(1)}^{5 / 2} \nabla_{\|} T_{D^{+}(2)} \\
q_{D^{+}(2)}= & -18.5 T_{D^{+}(2)}^{5 / 2} \nabla_{\|} T_{D^{+}(2)} \\
& -4.34 T_{D^{+}(2)}^{5 / 2} \nabla_{\|} T_{D^{+}(1)}
\end{aligned}
$$

Summing all contributions, one recovers the typical value $\kappa^{0}=57.2$ for deuterium when treated as a single species (see Equation B.2 in Appendix Appendix A). If this decomposition seems quite artificial for this splitting of deuterium in two, it would be of more interest when considering a mix of hydrogen isotopes, for instance a D-T mix. In that case, Zhdanov closure gives the coefficients linking the deuterium and tritium heat fluxes with deuterium temperature gradient and tritium temperature gradient contributions. If the two species show different temperature profiles, the heat flux computed by Zhdanov closure then differs from a simple Fourier law.

\section{Example of applications: JET D-T plasma}

\subsection{Simulation setting}

In this section, a typical application of multi-component plasma with hydrogen isotope mixture is presented: a JET D-T plasma. Moreover, we include also Nitrogen which is injected in the divertor to radiate in the edge and reduce the heat load at the strike points. 

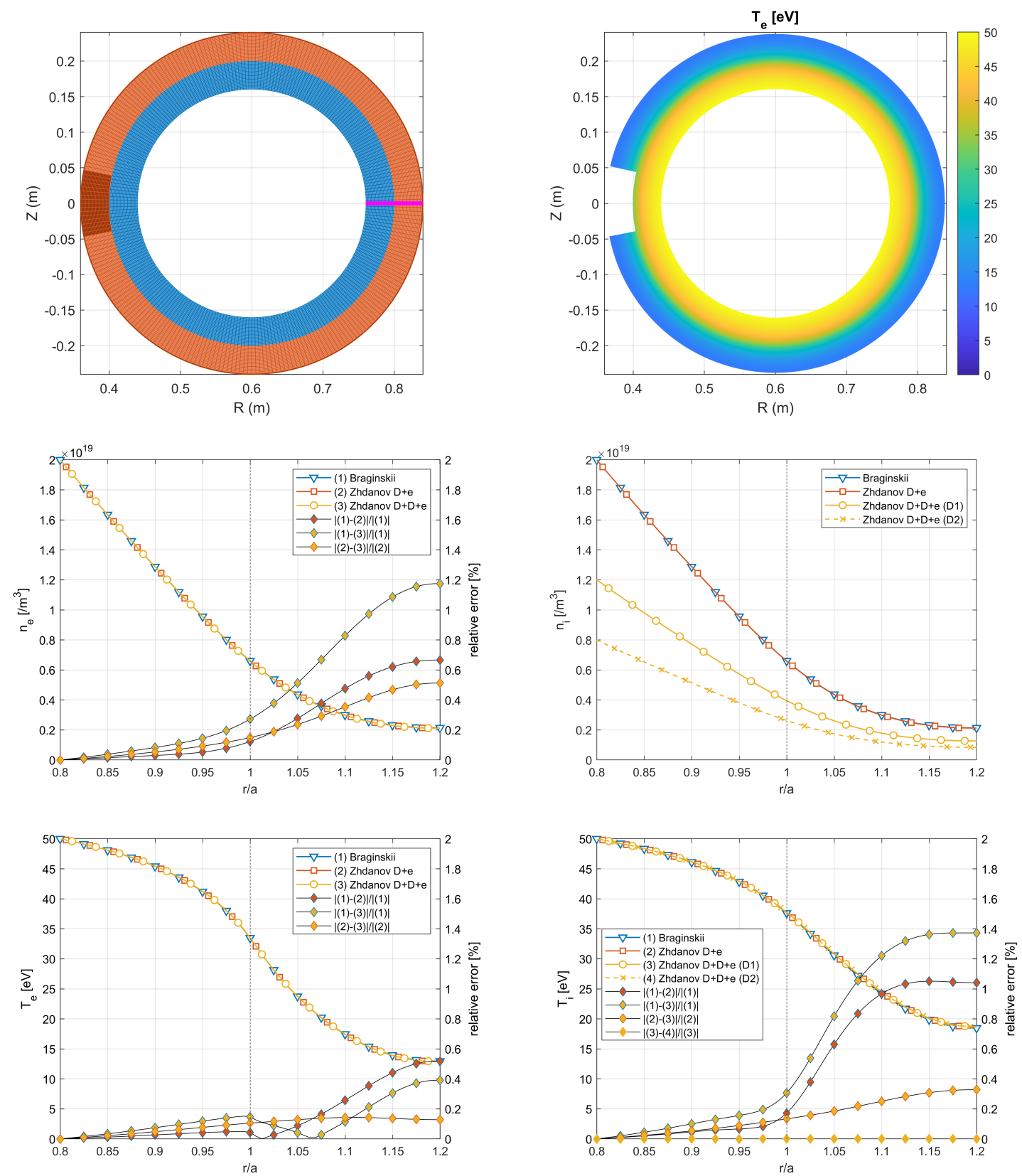

Figure 5. Inboard mid-plane profiles of electron and ion density and temperature. The three simulation setups are plotted with different colors.

As a reference magnetic equilibrium, we used JET shot number 97395 which is a high current $\left(I_{p}=3 \mathrm{MA}\right)$, high power $\left(P_{a d d}=P_{N B I}+P_{I C R H}=28+6=34 \mathrm{MW}\right)$ H-mode plasma scenario developped in 2017 in the framework of Eurofusion T17-07 experimental task "DT scenario extrapolation", experiment M15-01 "Baseline scenario". The SOLEDGE3X-EIRENE mesh of the magnetic equilibrium is plotted on Figure 6 where both SOLEDGE (quadrangles) and EIRENE (triangles) grid are shown. The radial extension in the core stops at $r / a=0.8$.

The following settings are used for the simulation. We prescribe midplane 

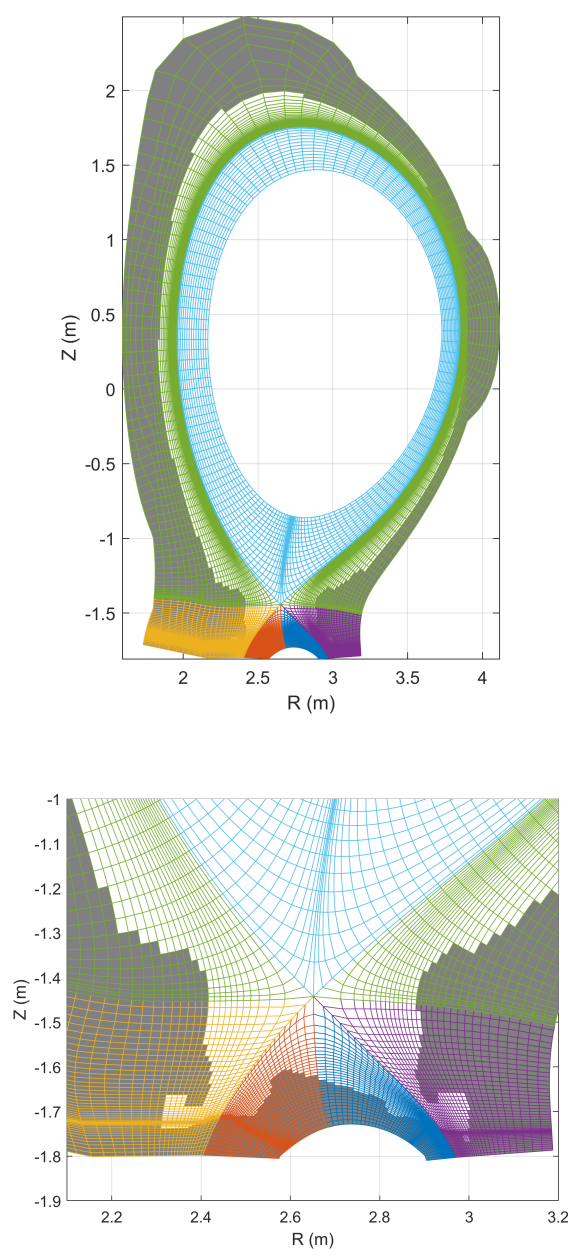
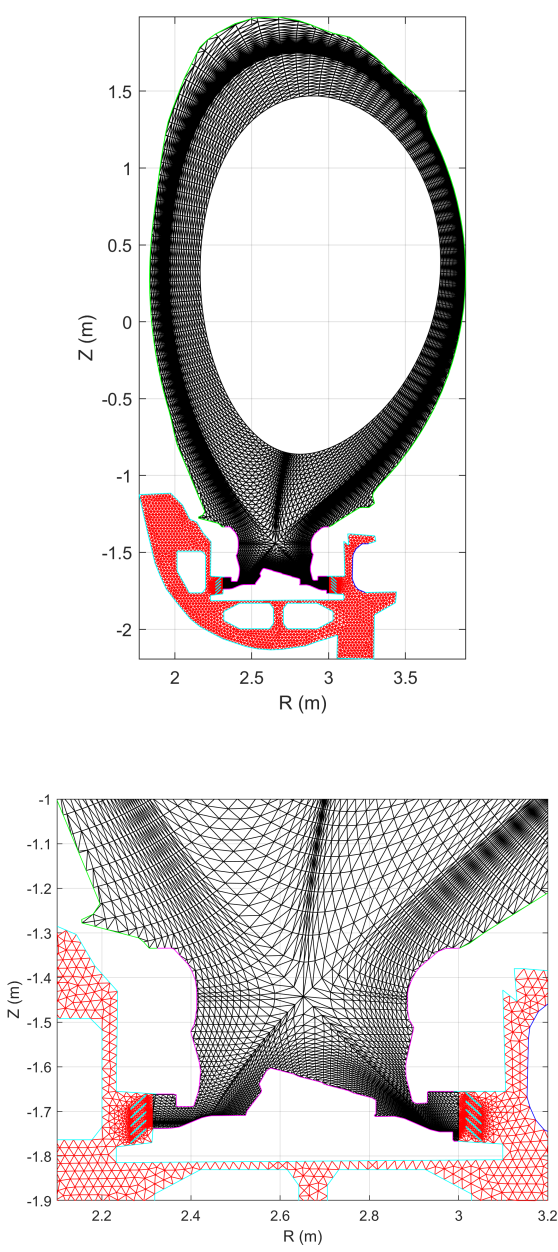

Figure 6. SOLEDGE3X-EIRENE mesh based on JET \#97395 equilibrium at $t=50 \mathrm{~s}$. Left: SOLEDGE3X mesh based on quadrangles grid extended to the wall. Right: EIRENE grid based on triangles. Wall types are indentified with different colors (green for Be, pink for $\mathrm{W}$ and dark blue for pumping surfaces, light blue for subdivertor wall).

profiles for Deuterium density, Deuterium temperature and electron temperature in the midplane. The code automatically adjust cross-field transport coefficients to match these input profiles which for our test case come from HRTS data from JET \#97395 where half electron density profile has been taken as a proxy for deuterium density profile, considering in our case a 50/50 D-T mix. Cross-field transport coefficients are assumed to be homogeneous on the flux surface (no poloidal dependence). Dirichlet boundary conditions are set at the core boundary to force "experimental" values for Deuterium and Tritium density as well as for electron temperature. For nitrogen, one imposes zero flux. Fueling in the edge is performed by Deuterium and Tritium gas puff near the inner strike point on high field side (HFS) vertical target (GIM 12). The gas puff rate is set to $10^{22} e^{-} \cdot s^{-1}$ for Deuterium and Tritium. Finally, we inject Nitrogen in the HFS private flux region (GIM 11) with a fixed puff rate $\varphi_{N}=1.4 \times 10^{22} e^{-} \cdot s^{-1}$. 
The location of gas puffs is plotted on Figure 9. The chamber wall is described by two different materials: a tungsten area in the divertor with a recycling coefficient set to 1 for Deuterium, Tritium, and Nitrogen, a Beryllium area on the main chamber wall with a recycling coefficient set to 0.99 for Deuterium, Tritium and Nitrogen. Pumping surfaces are located in the subdivertor at the location of cryo pumps; their recycling coefficient is set to 0.5 for Deuterium, Tritium and Nitrogen. The wall configuration is plotted on Figure 6.

Figure 7 shows the radial dependence of transport coefficients on the low field side. The well in diffusivity profiles near the separatrix implements the transport barrier

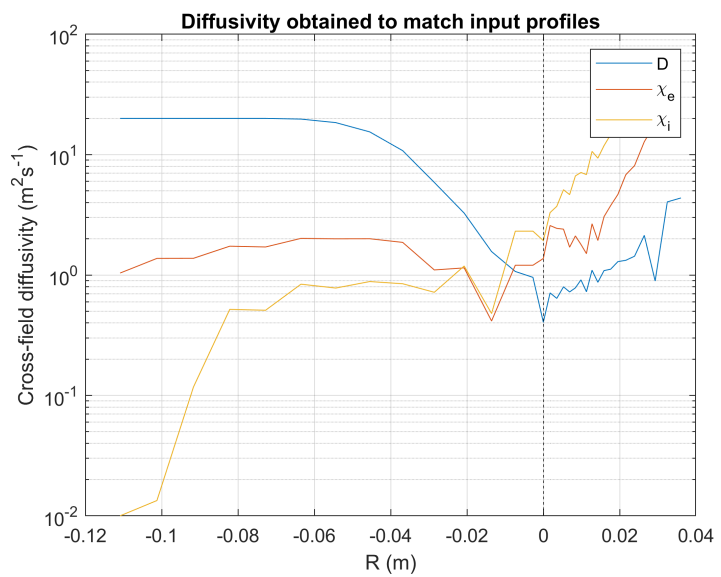

Figure 7. Transport coefficients used in JET D-T simulation. radial dependency at the LFS midplane.

characteristic of H-mode plasmas. One assumes all ion species share the same value for $D$ and $\chi$. One also assumes $\nu=D$. Drifts are switch-off. Figure 8 shows a comparison between simulation results and "experimental measurements" for main ions and electron density and temperatures in the edge plasma for our reference JET shot \#97395. The experimental profile for electron density was assumed to be shared between Deuterium and Tritium electrons (neglecting electrons from Nitrogen), hence the profile used as input for Deuterium and Tritium density. The code properly matches the input profile adjusting the profile for $D$ that is why $n_{D}+n_{T}$ profiles is in good agreeement with HRTS measurements. However, the $n_{e}$ profile computed by the code is higher to the HRTS data due to the extra electrons from Nitrogen. The $Z$ effective around the separatrix is found to be between around 3, see figure 9. The temperature profiles match more or less the input profiles and remain in the experimental range. Figure 10 shows electron density and temperature in the divertor. The plasma is partially detached on the high field side.

\subsection{Multi-component plasma analysis}

Despite identical fueling properties for Deuterium and Tritium (same rate at same location), identical recycling coefficient and identical radial transport coefficients, a 

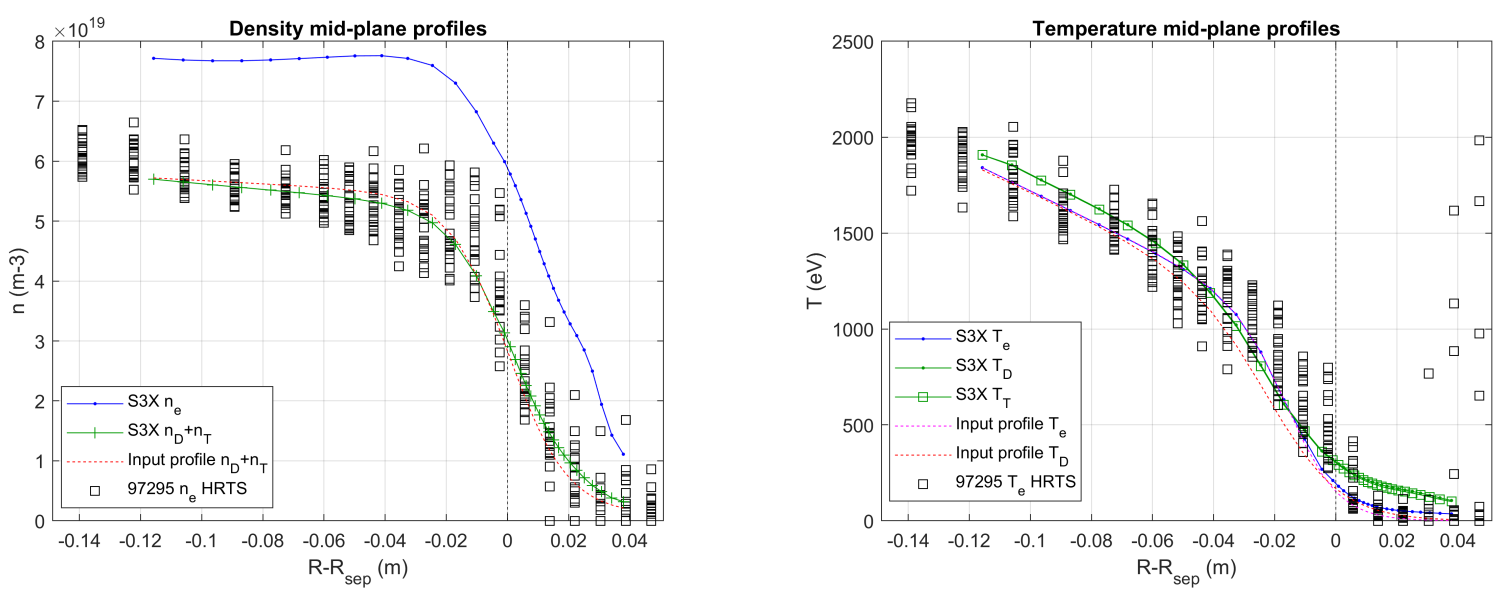

Figure 8. Comparison between simulated electron density and temperature at the LFS midplane with respect to experimental measurement in JET \#97395 (High resolution Thomson Scattering).

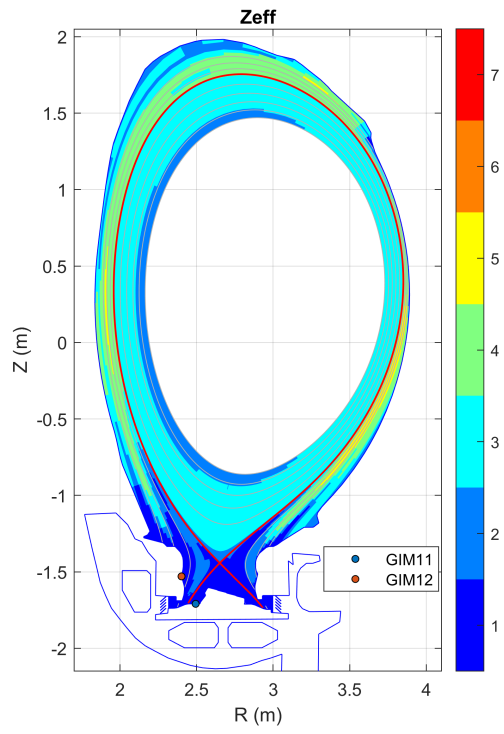

Figure 9. $\mathrm{Z}$ effective in the $\mathrm{D}+\mathrm{T}+\mathrm{N}$ H-mode simulation

significant discrepency can be found between the hydrogen isotopes radial profile in the divertor, see Figure 11. More precisely, a lower abundance of Tritium with respect to Deuterium is observed near the puff but a higher concentration of Tritium in the main SOL. One can expect a higher Scrape-off layer width $\lambda_{n}$ for tritium due to a lower sound speed for this isotop. Hence, the behavior observed in the present simulation could be very different if one uses a more complex boundary condition for the iosotop mixture (for example a common sound speed for the mixture). The extra abundance of Deuterium in the divertor is still under investigation. It must be noticed that the same albedo is used on the pump for Deuterium and Tritium. A better model could be implemented to match the different pumping efficiency between the two isotops. 

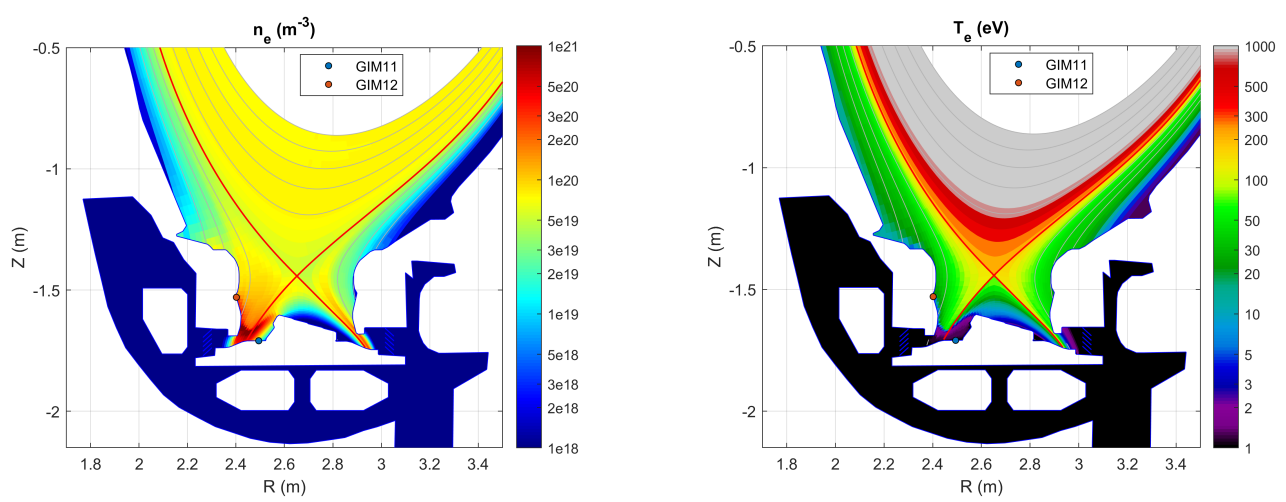

Figure 10. Left: electron density, right: electron temperature in the divertor.

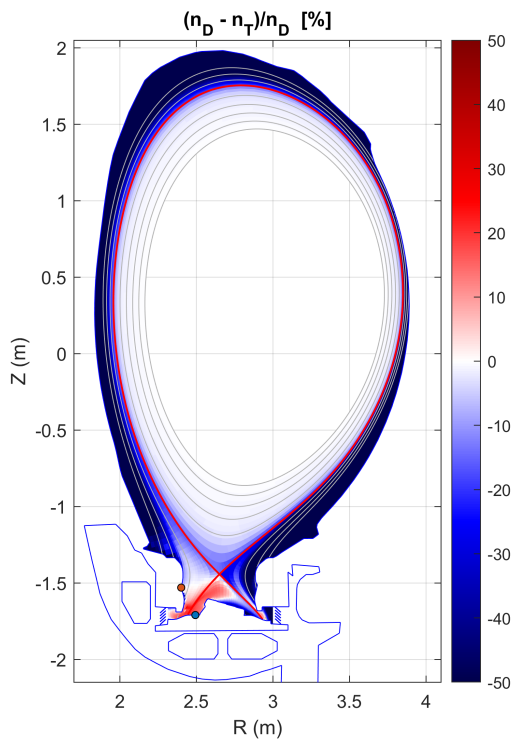

Figure 11. Relative difference between deuterium and tritium density

Radial profiles of the nitrogen ions density at the LFS midplane are plotted on Figure 12. One observes the presence of fully ionized Nitrogen $N^{7+}$ quite far in the scrape-off layer due to the slow recombination time compared with the radial transport time.

The SOLEDGE3X-EIRENE code also returns radiation maps from the different species. Several synthetic diagnostics are being implemented to help future comparison with experiments such as bolometry or spectroscopy [20].

\section{Conclusions}

Zhdanov closure has been rewritten in a matrix form and implemented in the SOLEDGE3X plasma code. It enables simulating complex multi-species plasma without resorting to the trace impurity assumption. The closure has been tested on simple 


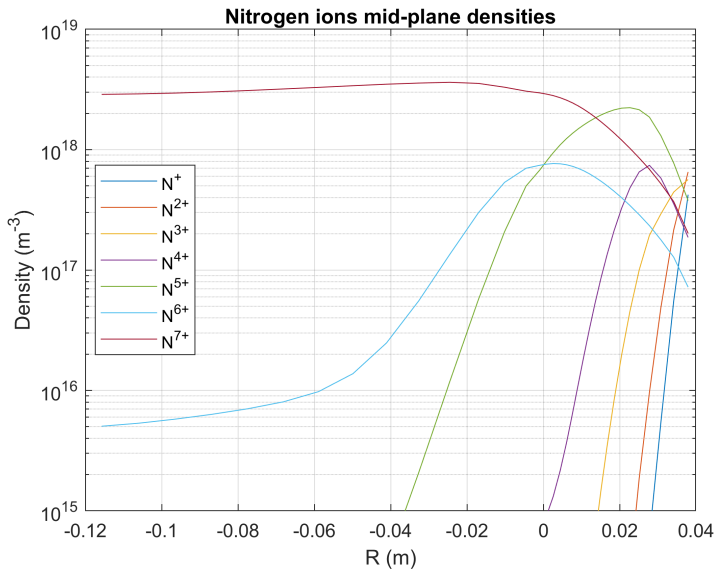

Figure 12. Radial profiles of plasma ion species at the outer midplane.
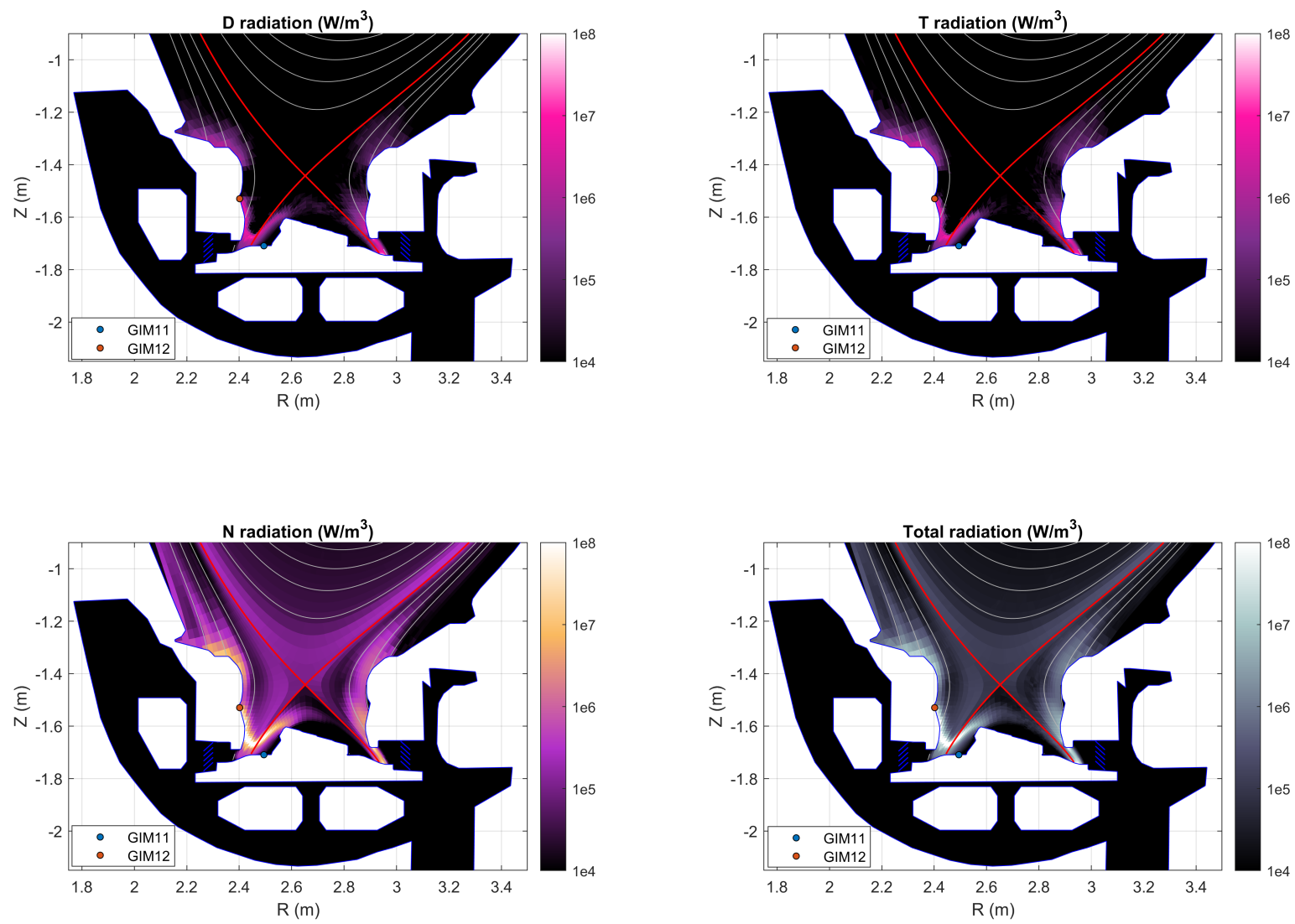

Figure 13. 2D maps of radiation for the different species.

plasma geometries and proves to be able to properly split a deuterium population in two. The collisional closure being constistent, the deuterium splitted plasma behaves the same way as a single deuterium species. The test was performed in $0 \mathrm{D}$ and in a circular 2D plasma. Finally, an example of application to a complex Deuterium+Tritium 
plasma on JET including Nitrogen seeding illustrates the typical application of such a closure to simulate multi-component plasma.

\section{Acknowledgements}

This work was granted access to the HPC resources of CINES, under the allocation 2021A0100510482 made by GENCI. Centre de calcul intensif d'Aix-Marseille is acknowledged for granting access to its high performance computing resources. This work was supported by the EoCoE-II project, grant agreement 824158, funded within the EUs H2020 program. This work has been carried out within the framework of the EUROfusion Consortium and has received funding from the Euratom research and training programme 2014-2018 and 2019-2020 under grant agreement No 633053. The views and opinions expressed herein do not necessarily reflect those of the European Commission

\section{Bibliography}

[1] V. M. Zhdanov. Transport Processes in Multicomponent Plasma. Taylor \& Francis, 2002.

[2] H. Bufferand et al. Numerical modelling for divertor design of west device with a focus on plasmawall interactions. Nucl. Fusion, 55, 2015.

[3] S. Wiesen et al. The new solps-iter code package. J. Nucl. Mat., 463, 2015.

[4] A.E. Jaervinen et al. Interpretation of radiative divertor studies with impurity seeding in type-i elmy h-mode plasmas in jet-ilw using edge2d-eirene. J. Nucl. Mat., 463, 2015.

[5] P. Tamain et al. The tokam3x code for the edge turbulence fluid simularions of tokamak plasmas in versatile magnetic geometries. J. Comp. Phys., 321, 2016.

[6] B.D. Dudson et al. Bout++: Recent and current developments. J. Plasma Phys., 81-1, 2014.

[7] A. Stegmeir et al. Grillix: a 3d turbulence code based on the flux-coordinate independent approach. Plasma Phys. Control. Fusion, 60, 2018.

[8] S.I. Braginskii. Transport processes in a plasma. Rev. of Plasma Phys., 1, 1965.

[9] H. Bufferand et al. Three-dimensional modelling of edge multi-component plasma taking into account realistic wall geometry. Nucl. Mat. Energy, 2019.

[10] E. Sytova et al. Impact of a new general form of friction and thermal forces on solps-iter modelling results. Contrib. Plasma Phys., 2018.

[11] V. Rozhansky et al. Momentum balance for impurities in solps transport code. J. Nucl. Mat., 463, 2015.

[12] A. Paredes et al. A penalization technique to model plasma facing components in a tokamak with temperature variations. J. Comp. Phys., 274, 2014.

[13] H. Bufferand and et al. Near wall plasma simulation using penalization technique with the transport code soledge2d-eirene. J. Nuclear Mat., 438, 2013.

[14] H. Bufferand et al. Implementation of drift velocities and currents in soledge2d-eirene. Nucl. Mat. Energy, 12, 2016.

[15] S. Baschetti, H. Bufferand, G. Ciraolo, N. Fedorczak, Ph. Ghendrih, E. Serre, and P. Tamain. Optimization of turbulence reduced model free parameters based on l-mode experiments and $2 \mathrm{~d}$ transport simulations. Contrib. Plasma Phys., 58, 2018.

[16] D. Tskhakaya. One-dimensional plasma sheath model in front of the divertor plates. Plasma Phys. Control. Fusion, 59, 2017.

[17] Peter Stangeby. The Plasma Boundary of Magnetic Fusion Devices. IoP, 2000. 
[18] H. Bufferand. Development of a fluid code for tokamak edge plasma simulation. Investigation on non-local transport. PhD thesis, Aix-Marseille Université, 2012.

[19] B.D. Dudson et al. Verification of bout++ by the method of manufactured solutions. Phys. Plasmas, 23, 2016.

[20] G. Ciraolo et al. First modeling of strongly radiating west plasmas with soledge-eirene. Nucl. Mat. Energy, 20, 2019.

[21] M. Raghunathan, Y. Marandet, H. Bufferand, G. Ciraolo, Ph Ghendrih, P. Tamain, and E. Serre. Generalized collisional fluid theory for multi-component, multi-temperature plasma using the linearized Boltzmann collision operator for scrape-off layer/edge applications. Plasma Physics and Controlled Fusion, 63(6):064005, May 2021. Publisher: IOP Publishing.

\section{Appendix A. Collision coefficients}

Appendix A.1. $\bar{G}_{\alpha \beta}^{(n)}$ coefficients

The coefficients $\bar{G}_{\alpha \beta}^{(n)}$ used in Zhdanov system of Equations 22 and 23 are given by:

$$
\bar{G}_{\alpha \beta}^{(n)}=\sum_{Z, \zeta} \bar{G}_{\alpha Z \beta \zeta}^{(n)}
$$

with

$$
\begin{aligned}
& \bar{G}_{\alpha Z \beta \zeta}^{(1)}=-\lambda_{\alpha Z \beta \zeta} \\
& \bar{G}_{\alpha Z \beta \zeta}^{(2)}=\frac{3}{5} \lambda_{\alpha Z \beta \zeta} \\
& \bar{G}_{\alpha Z \beta \zeta}^{(3)}=-2\left(1+\frac{3}{5} \frac{m_{\beta}}{m_{\alpha}}\right) \lambda_{\alpha Z \beta \zeta} \\
& \bar{G}_{\alpha Z \beta \zeta}^{(4)}=\frac{4}{5} \lambda_{\alpha Z \beta \zeta} \\
& \bar{G}_{\alpha Z \beta \zeta}^{(5)}=-\left(\frac{13}{10} \frac{m_{\beta}}{m_{\alpha}}+\frac{8}{5}+3 \frac{m_{\alpha}}{m_{\beta}}\right) \kappa_{\alpha \beta} \lambda_{\alpha Z \beta \zeta} \\
& \bar{G}_{\alpha Z \beta \zeta}^{(6)}=\frac{27}{10} \kappa_{\alpha \beta} \lambda_{\alpha Z \beta \zeta} \\
& \bar{G}_{\alpha Z \beta \zeta}^{(8)}=-\frac{3}{14} \lambda_{\alpha Z \beta \zeta} \\
& \bar{G}_{\alpha Z \beta \zeta}^{(9)}=\frac{3}{5}\left(\frac{23}{28} \frac{m_{\beta}}{m_{\alpha}}+\frac{8}{7}+3 \frac{m_{\alpha}}{m_{\beta}}\right) \kappa_{\alpha \beta} \lambda_{\alpha Z \beta \zeta} \\
& \bar{G}_{\alpha Z \beta \zeta}^{(10)}=-\frac{45}{28} \kappa_{\alpha \beta} \lambda_{\alpha Z \beta \zeta} \\
& \bar{G}_{\alpha Z \beta \zeta}^{(11)}=-\left[\frac{433}{280}\left(\frac{m_{\beta}}{m_{\alpha}}\right)^{2}+\frac{136}{35} \frac{m_{\beta}}{m_{\alpha}}+\frac{459}{35}\right. \\
& \left.+\frac{32}{5} \frac{m_{\alpha}}{m_{\beta}}+5\left(\frac{m_{\alpha}}{m_{\beta}}\right)^{2}\right] \kappa_{\alpha \beta}^{2} \lambda_{\alpha Z \beta \zeta} \\
& \bar{G}_{\alpha Z \beta \zeta}^{(12)}=\frac{75}{8} \kappa_{\alpha \beta}^{2} \lambda_{\alpha Z \beta \zeta} \\
& \bar{G}_{\alpha Z \beta \zeta}^{(13)}=\left(\frac{18}{35} \frac{m_{\beta}}{m_{\alpha}}+\frac{6}{5}\right) \lambda_{\alpha Z \beta \zeta}
\end{aligned}
$$


Implementation of multi-component Zhdanov closure in SOLEDGE3X

$$
\begin{aligned}
\bar{G}_{\alpha Z \beta \zeta}^{(14)} & =-\frac{24}{35} \lambda_{\alpha Z \beta \zeta} \\
\bar{G}_{\alpha Z \beta \zeta}^{(15)} & =-\left(\frac{51}{35}\left(\frac{m_{\beta}}{m_{\alpha}}\right)^{2}+\frac{37}{7} \frac{m_{\beta}}{m_{\alpha}}+\frac{22}{5}+4 \frac{m_{\alpha}}{m_{\beta}}\right) \kappa_{\alpha \beta} \lambda_{\alpha Z \beta \zeta} \\
\bar{G}_{\alpha Z \beta \zeta}^{(16)} & =\frac{24}{7} \frac{m_{\beta}}{m_{\alpha}} \kappa_{\alpha \beta} \lambda_{\alpha Z \beta \zeta}
\end{aligned}
$$

where

$$
\lambda_{\alpha Z \beta \zeta}=\frac{1}{3}(2 \pi)^{-3 / 2} n_{\alpha Z} n_{\beta \zeta} e^{4} Z^{2} \zeta^{2} \mu_{\alpha \beta}^{1 / 2} \frac{\ln \Lambda_{\alpha \beta}}{\left(k_{B} T\right)^{3 / 2} \varepsilon_{0}^{2}}
$$

the Coulomb coefficient being given by

$$
\Lambda_{\alpha \beta}=\frac{12 \pi \varepsilon_{0}^{3 / 2} k T}{Z_{\mathrm{eff}} e^{2}}\left[\frac{k_{B} T}{n_{e} e^{2}\left(1+Z_{\mathrm{eff}}\right)}\right]^{1 / 2}
$$

Also

$$
\begin{aligned}
\mu_{\alpha \beta} & =\frac{m_{\alpha} m_{\beta}}{m_{\alpha}+m_{\beta}} \\
\kappa_{\alpha \beta} & =\frac{m_{\alpha} m_{\beta}}{\left(m_{\alpha}+m_{\beta}\right)^{2}} .
\end{aligned}
$$

Notice the differences in the coefficients for $\bar{G}_{\alpha Z \beta \zeta}^{(14)}$ and $\bar{G}_{\alpha Z \beta \zeta}^{(16)}$, also mentioned in Ref. [21].

\section{Appendix A.2. Collision times}

The collision time between species $(\alpha, Z)$ and $(\beta, \zeta)$ is given by:

$$
\tau_{\alpha Z \beta \zeta}^{-1}=\frac{16 \pi^{1 / 2}}{3} n_{\beta}\left(\frac{\gamma_{\alpha Z \beta \zeta}}{2}\right)^{3 / 2}\left(\frac{Z \zeta e^{2}}{4 \pi \varepsilon_{0} \mu_{\alpha \beta}}\right)^{2} \ln \Lambda_{\alpha \beta}
$$

where

$$
\gamma_{\alpha Z \beta \zeta}=\frac{\gamma_{\alpha Z} \gamma_{\beta \zeta}}{\gamma_{\alpha Z}+\gamma_{\beta \zeta}}
$$

with

$$
\gamma_{\alpha Z}=\frac{m_{\alpha}}{k_{B} T_{\alpha Z}}
$$

The collision time averaged over ionization states:

$$
\tau_{\alpha \beta}^{-1}=\sum_{Z, \zeta} \frac{n_{\alpha Z} \tau_{\alpha Z \beta \zeta}^{-1}}{n_{\alpha}}
$$

At last, the averaged collision time of species $\alpha$ :

$$
\tau_{\alpha}^{-1}=\sum_{\beta} \frac{\mu_{\alpha \beta}}{m_{\alpha}} \tau_{\alpha \beta}^{-1}
$$


Appendix A.3. The $S_{\alpha}^{(n)}$ and $c_{\alpha}^{(n)}$ coefficients

The coefficients denoted $S_{\alpha}^{(n)}$ by Zhdanov in [1] are given by

$$
\begin{aligned}
S_{\alpha}^{(2)} & =\frac{5}{2} \sum_{\beta} \frac{\mu_{\alpha \beta}}{m_{\alpha}} \bar{G}_{\alpha \beta}^{(2)} \\
S_{\alpha}^{(5)} & =\sum_{\beta} \bar{G}_{\alpha \beta}^{(5)} \\
S_{\alpha}^{(8)} & =\frac{35}{2} \sum_{\beta}\left(\frac{\mu_{\alpha \beta}}{m_{\alpha}}\right)^{2} \bar{G}_{\alpha \beta}^{(8)} \\
S_{\alpha}^{(9)} & =\sum_{\beta} \frac{\mu_{\alpha \beta}}{m_{\alpha}} \bar{G}_{\alpha \beta}^{(9)} \\
S_{\alpha}^{(11)} & =\sum_{\beta} \bar{G}_{\alpha \beta}^{(11)} \\
c_{\alpha}^{(5)} & =\frac{5}{2} \frac{\tau_{\alpha} \tau_{\alpha \alpha}^{-1} S_{\alpha}^{(11)}}{D_{\alpha}} \\
c_{\alpha}^{(6)} & =\frac{S_{\alpha}^{(2)} S_{\alpha}^{(11)}-S_{\alpha}^{(8)} S_{\alpha}^{(9)}}{D_{\alpha}}
\end{aligned}
$$

where

$$
D_{\alpha}=S_{\alpha}^{(5)} S_{\alpha}^{(11)}-7\left(S_{\alpha}^{(9)}\right)^{2}
$$

\section{Appendix B. Comparison between Deuterium+electrons and Deuterium+Deuterium+electrons plasma}

We report here the numerical value found applying Zhdanov closure to either a simple deuterons + electrons plasma or the same plasma where the deuteron population is splitted into two "classes" to make a deuterons (type 1) +deuterons (type 2) + electrons plasma. The properties of the populations are summarized in table B1.

\begin{tabular}{c|cccccc} 
case & $n_{e}\left[m^{-3}\right]$ & $n_{D 1}\left[m^{-3}\right]$ & $n_{D 2}\left[m^{-3}\right]$ & $T_{e}[e V]$ & $T_{D 1}[e V]$ & $T_{D 2}[\mathrm{eV}]$ \\
\hline 1 & $2 e 19$ & $2 e 19$ & & 50 & 50 & \\
2 & $2 e 19$ & $1.2 e 19$ & $0.8 e 19$ & 50 & 50 & 50
\end{tabular}

Table B1. Properties of the two plasmas considered for comparison. The two plasmas are virtually identical.

For the first case, the matrix linking heat fluxes, friction forces with temperature gradients and velocities takes the numerical values given by Equations B.1 and B.2 .

$$
\left(\begin{array}{c}
q_{\|, e} \\
q_{\|, D} \\
R_{\|, e} \\
R_{\|, D}
\end{array}\right)=M_{D e} \cdot\left(\begin{array}{c}
\nabla_{\|} T_{e} \\
\nabla_{\|} T_{D} \\
v_{\|, e} \\
v_{\|, D}
\end{array}\right)
$$


where

$$
M_{D e}=\left(\begin{array}{cccc}
-2237 T_{e}^{5 / 2} & 0 & 3.22 e n_{e} T_{e} & -0.71 e n_{e} T_{e} \\
0 & -57.2 T_{D}^{5 / 2} & 0 & 2.5 e n_{D} T_{D} \\
-0.71 e n_{e} & 0 & -0.51 m_{e} n_{e} \nu_{e} & 0.51 m_{e} n_{e} \nu_{e} \\
0.71 e n_{e} & 0 & 0.51 m_{e} n_{e} \nu_{e} & -0.51 m_{e} n_{e} \nu_{e}
\end{array}\right)
$$

One recovers the typical values of the Braginskii's closure.

For the second case, the matrix linking heat fluxes, friction forces with temperature gradients and velocities takes the numerical values given by Equation B.3 and B.4.

$$
\left(\begin{array}{c}
q_{\|, e} \\
q_{\|, D 1} \\
q_{\|, D 2} \\
R_{\|, e} \\
R_{\|, D 1} \\
R_{\|, D 2}
\end{array}\right)=M_{D D e} \cdot\left(\begin{array}{c}
\nabla_{\|} T_{e} \\
\nabla_{\|} T_{D 1} \\
\nabla_{\|} T_{D 2} \\
v_{\|, e} \\
v_{\|, D 1} \\
v_{\|, D 2}
\end{array}\right)
$$

where

$$
M_{D D e}=\left(\begin{array}{cccccc}
-2237 T_{e}^{5 / 2} & 0 & 0 & 3.22 e n_{e} T_{e} & -0.43 e n_{e} T_{e} & -0.28 e n_{e} T_{e} \\
0 & -30.0 T_{D 1}^{5 / 2} & -4.34 T_{D 1}^{5 / 2} & 0 & 2.72 e n_{D 1} T_{D 1} & -0.22 e n_{D 1} T_{D 1} \\
0 & -4.34 T_{D 2}^{5 / 2} & -18.5 T_{D 2}^{5 / 2} & 0 & -0.33 e n_{D 2} T_{D 1} & 2.83 e n_{D 2} T_{D 2} \\
-0.71 e n_{e} & 0 & 0 & -0.51 m_{e} n_{e} \nu_{e} & 0.31 m_{e} n_{e} \nu_{e} & 0.2 m_{e} n_{e} \nu_{e} \\
0.43 e n_{e} & -0.13 e n_{e} & 0.13 e n_{e} & 0.31 m_{e} n_{e} \nu_{e} & -9.2 m_{e} n_{e} \nu_{e} & 8.9 m_{e} n_{e} \nu_{e} \\
0.28 e n_{e} & 0.13 e n_{e} & -0.13 e n_{e} & 0.21 m_{e} n_{e} \nu_{e} & 8.9 m_{e} n_{e} \nu_{e} & -9.1 m_{e} n_{e} \nu_{e}
\end{array}\right)(
$$

In order to compare the two cases, if the plasma where deuterons are splitted into two populations behave the same as the single deuteron case, one has for all intensive quantities:

$$
\begin{aligned}
& \nabla_{\|, D}=\nabla_{\|, D 1}=\nabla_{\|, D 2} \\
& v_{\|, D}=v_{\|, D 1}=v_{\|, D 2}
\end{aligned}
$$

and for all extensive quantities:

$$
\begin{aligned}
& q_{\|, D}=q_{\|, D 1}+q_{\|, D 2} \\
& R_{\|, D}=R_{\|, D 1}+R_{\|, D 2}
\end{aligned}
$$

The matrix $P$ given by

$$
P=\left(\begin{array}{llllll}
1 & 0 & 0 & 0 & 0 & 0 \\
0 & 1 & 1 & 0 & 0 & 0 \\
0 & 0 & 0 & 1 & 0 & 0 \\
0 & 0 & 0 & 0 & 1 & 1
\end{array}\right)
$$


makes the link between the two cases following

$$
\left(\begin{array}{c}
q_{\|, e} \\
q_{\|, D} \\
R_{\|, e} \\
R_{\|, D}
\end{array}\right)=P \cdot\left(\begin{array}{c}
q_{\|, e} \\
q_{\|, D 1} \\
q_{\|, D 2} \\
R_{\|, e} \\
R_{\|, D 1} \\
R_{\|, D 2}
\end{array}\right) \text { and }\left(\begin{array}{c}
\nabla_{\|} T_{e} \\
\nabla_{\|} T_{D 1} \\
\nabla_{\|} T_{D 2} \\
v_{\|, e} \\
v_{\|, D 1} \\
v_{\|, D 2}
\end{array}\right)=P^{t} \cdot\left(\begin{array}{c}
\nabla_{\|} T_{e} \\
\nabla_{\|} T_{D} \\
v_{\|, e} \\
v_{\|, D}
\end{array}\right)(
$$

If Zhdanov closure is consistent, a reduction of the "splitted deuterons" case into a "single deuteron" case can be computed as

$$
\widetilde{M_{D e}}=P \cdot M_{D D e} \cdot P^{t}
$$

The two approaches are compared computing the relative difference between the two matrices:

$$
\varepsilon=\max _{i, j}\left|\frac{{\widetilde{M_{D e}}}^{i j}-M_{D e}^{i j}}{M_{D e}^{i j}}\right|
$$

In our case, we found $\varepsilon \approx 10^{-11}$ which indicates a almost perfect agreement between the two cases. 